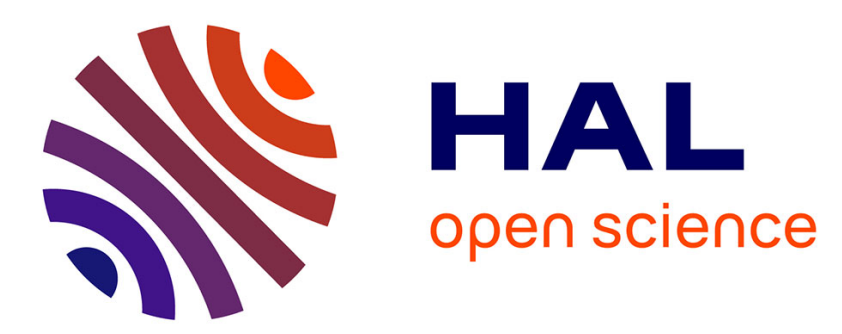

\title{
Diameter Determination on Restricted Graph Families
}

Derek G. Corneil, Feodor F. Dragan, Michel Habib, Christophe Paul

\section{To cite this version:}

Derek G. Corneil, Feodor F. Dragan, Michel Habib, Christophe Paul. Diameter Determination on Restricted Graph Families. Discrete Applied Mathematics, 2001, 113 (2-3), pp.146-166. 10.1016/S0166218X(00)00281-X . lirmm-00090363

\section{HAL Id: lirmm-00090363 \\ https://hal-lirmm.ccsd.cnrs.fr/lirmm-00090363}

Submitted on 30 Aug 2006

HAL is a multi-disciplinary open access archive for the deposit and dissemination of scientific research documents, whether they are published or not. The documents may come from teaching and research institutions in France or abroad, or from public or private research centers.
L'archive ouverte pluridisciplinaire HAL, est destinée au dépôt et à la diffusion de documents scientifiques de niveau recherche, publiés ou non, émanant des établissements d'enseignement et de recherche français ou étrangers, des laboratoires publics ou privés. 


\title{
Diameter Determination on Restricted Graph Families $\S$
}

\author{
Derek G. CorneIL ${ }^{*} \quad$ Feodor F. Dragan ${ }^{\dagger}$ Michel HaBIB ${ }^{\ddagger}$ \\ Christophe PAUL $\ddagger$
}

\begin{abstract}
Determining the diameter of a graph is a fundamental graph operation, yet no efficient (i.e. linear or quadratic time) algorithm is known. In this paper, we examine the diameter problem on chordal graphs and ATfree graphs and show that a very simple (linear time) 2-sweep LexBFS algorithm identifies a vertex of maximum eccentricity unless the given graph has a specified induced subgraph (it was previously known that a single LexBFS algorithm is guaranteed to end at a vertex that is within 1 of the diameter for chordal graphs and AT-free graphs). As a consequence of the forbidden induced subgraph result on chordal graphs, our algorithm is guaranteed to work optimally for directed path graphs (it was previously known that a single LexBFS algorithm is guaranteed to work optimally for interval graphs).
\end{abstract}

\section{Introduction}

Recently considerable attention has been given to the problem of developing fast and simple algorithms for various classical graph problems. The motivation for such algorithms stems from our need to solve these problems on very large input graphs, thus the algorithms must be not only fast, but also easily implementable. Determining a graph diameter is a classical and well-known problem.

For arbitrary graphs (with $n$ vertices and $m$ edges), as well as for various restricted graph families, the current fastest algorithm for this problem achieves the time bound of $O(\mathrm{~nm})$ (see for example [24]) which is too slow to be practical for very large graphs. This naive algorithm performs Breadth First Searches (BFSs) from each vertex of the graph and actually finds the whole distance matrix of the graph. For dense graphs, the best result known is by Seidel [28], who showed that the distance matrix (and hence the diameter) of a graph can be computed in $O(M(n) \log n)$ time where $M(n)$ denotes the time complexity for matrix multiplication involving small integers only. (Currently, $M(n)$ is known to be $O\left(n^{2.376}\right)$ [6].) Note also that in a recent paper [1], the authors solve the all pairs shortest path problem with an additive error at most 2 without matrix multiplication in $O\left(n^{2.5} \sqrt{\log n}\right)$ time. They obtain also a ratio $2 / 3$ approximation to the diameter in time $O\left(m \sqrt{n \log n}+n^{2} \log n\right)$.

\footnotetext{
*Dept. of Computer Science, University of Toronto, Toronto M5S 3G4, Canada

$\dagger$ Dept. of Computer Science, University of California at Los Angeles, 3514 Boelter Hall, Los Angeles, CA 90095-1596 USA

‡LIRMM, UMR CNRS-Université Montpellier II, 161 rue Ada, 34392 Montpellier cedex 5, France

$\S$ An extended abstract of these results has been presented at WG'98 [7]
} 
Algorithms of complexity $O\left(n^{2}\right)$ for computing the distance matrix (and hence the diameter) in different particular graph classes were presented in $[10$, $2,4,20]$. Linear-time algorithms for computing the diameter, that avoid the computation of the whole distance matrix, have been designed for trees [21], maximal outerplanar graphs [16], interval graphs [25, 14], ptolemaic graphs [14], strongly chordal graphs, dually chordal graphs [2], distance-hereditary graphs $[11,13]$ and for graphs of benzenoid systems [4]. It is not yet clear for general graphs whether computing the diameter is easier than computing the whole distance matrix.

In this paper, we study the problem of determining a vertex of high eccentricity for chordal graphs and AT-free graphs. The eccentricity of a vertex $x$ is $e c c(x)=\max _{y \in V} d(x, y)$, where $d(x, y)$ denotes the distance between $x$ and $y$. The diameter of a graph equals the maximum eccentricity achieved by any vertex in the graph. Given $v$, a vertex of maximum eccentricity, it is trivial to determine the set of vertices whose distance from $v$ equals the diameter of $G$ (these vertices constitute the last layer of a BFSfrom $v$ ).

A graph is chordal iff there is no chordless cycle of length more than 3. It is well-known that chordal graphs are exactly the intersection graphs of subtrees in trees $[3,17]$. Interval graphs can be defined as the intersection graphs of subpaths in paths (see [23]). A natural generalization of interval graphs is the concept of directed path graphs. A graph is a directed path graph iff it is the intersection graph of a collection of directed paths in a rooted directed tree [18]. A chord $x_{i} x_{j}$ in a cycle $C=\left(x_{1}, x_{2}, \ldots, x_{2 n}\right)$ is an odd chord if, in $C$, $d\left(x_{i}, x_{j}\right)$ is odd. A graph is strongly chordal [15] if it is chordal and each cycle of even length at least 6 has an odd chord. Strongly chordal graphs represent an interesting subclass of chordal graphs which includes directed path graphs. Three vertices $u, v, w$ are an asteroidal triple (AT) if between any two of them, there exists a path that avoids the neighbourhood of the remaining vertex. A graph is AT-free if it does not contain an AT. The famous characterization of interval graphs given by Lekkerkerker and Boland says that a graph is interval if and only if it is chordal and AT-free [23].

The algorithm that we present involves two sweeps of the well-known Lexicographic Breadth First Search (LexBFS) introduced by Rose, Tarjan and Lueker [27] (see algorithm 1) for the recognition of chordal graphs. An example of a LexBFS sweep is presented in figure 13. It is somewhat surprising that LexBFS seems to play a fundamental role for both chordal and AT-free graphs, two families that exhibit very little structural similarity (see for example $[8,9,17,26,27])$. Dragan et al [14] and Dragan [12] have proved the following theorem that demonstrates further similar behaviour for chordal and AT-free graphs.

Theorem 1 [14, 12] Let $v$ be the vertex visited last by an arbitrary LexBFS. If the graph is chordal or AT-free, then the eccentricity of $v$ is within 1 of the diameter of the graph. If the graph is interval, the eccentricity of $v$ equals the diameter. 


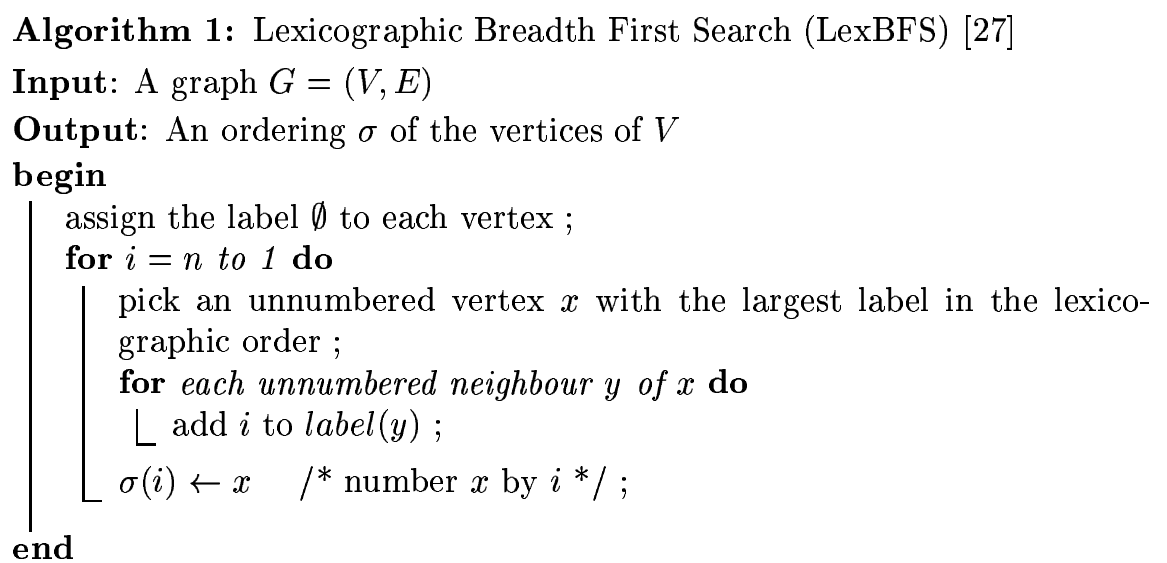

Note that LexBFS can be started from any vertex of the graph $G$. We will denote by LexBFS $(w)$ a LexBFS started from vertex $w$. In this paper, we examine the following very simple 2 -sweep LexBFS algorithm and study its performance on chordal and AT-free graphs.

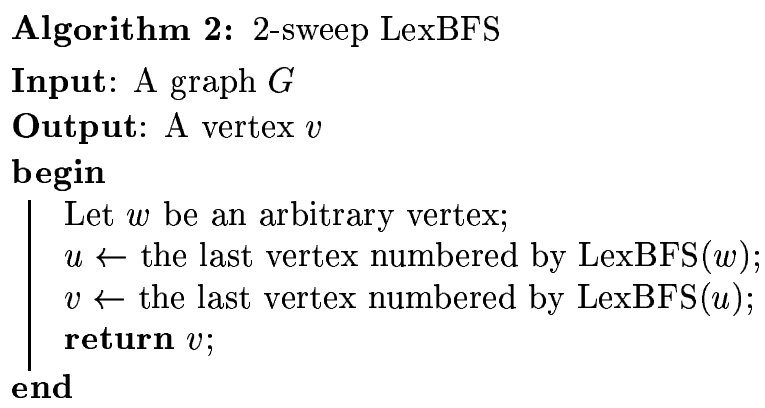

In particular, we examine conditions when $\operatorname{ecc}(v)=\operatorname{diam}(G)-1$, where $v$ is the vertex returned by the 2 -sweep algorithm. These conditions include forbidden subgraph results for both chordal and AT-free graphs. The forbidden subgraph result for chordal graphs immediately shows that the algorithm works optimally (i.e. $\operatorname{ecc}(v)=\operatorname{diam}(G)$ ) for directed path graphs.

Before presenting these results, we show that it is unlikely that the diameter problem on either chordal or AT-free graphs can be solved in quadratic time. To do this we introduce the disjoint sets problem.

\section{Disjoint Sets Problem}

Given $\mathcal{S}=\left\{S_{1}, S_{2}, \ldots, S_{n}\right\}$ sets over the base set $\mathcal{X}$, the Disjoint Sets Problem (DSP) asks whether there exist $i$ and $j$ such that $S_{i} \cap S_{j}=\emptyset$. As pointed out by Chepoi and Dragan in [5], a fast algorithm (i.e. quadratic time or better) for determining whether a split graph (and thus a chordal graph) has diameter 2 or 3 would imply a fast algorithm for the DSP (see figure 1). 


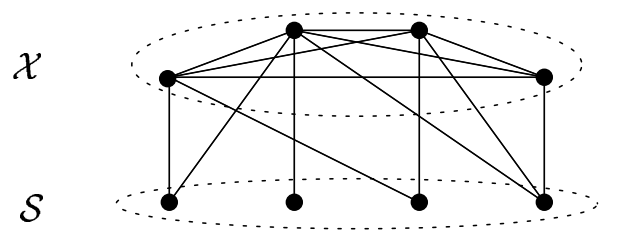

Figure 1: The set $\mathcal{X}$ is represented by a clique and $\mathcal{S}$ by an independent set. A set $S_{i}$ is adjacent to its elements in $\mathcal{X}$. The diameter of this graph is 3 iff there exist two disjoint sets in $\mathcal{S}$.

In figure 2, a similar transformation is presented to show that the diameter equals 2 or 3 problem on co-comparability graphs (and thus AT-free graphs) would have the same impact on the DSP.

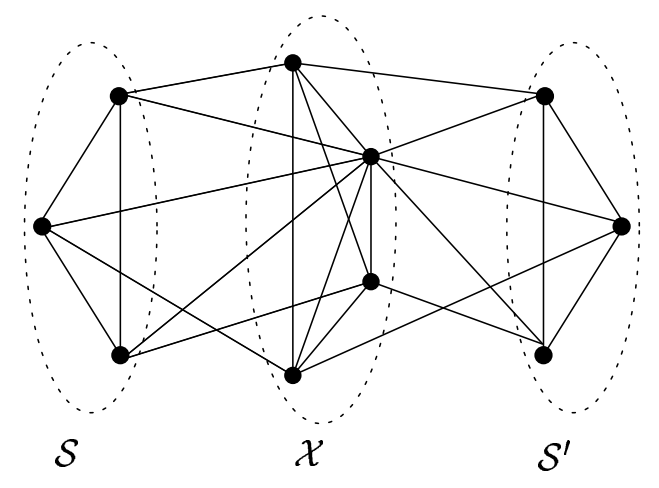

Figure 2: The set $\mathcal{X}$ is represented by a clique. Two copies of $\mathcal{S}$ are also represented by two cliques. A set $S_{i}$ (resp. $S_{i}^{\prime}$ ) is adjacent to its elements in $\mathcal{X}$. The diameter of this graph is 3 iff there exist two disjoint sets in $\mathcal{S}$.

Thus it seems unlikely that a linear or quadratic time algorithm exists for the diameter problem on either chordal or AT-free graphs. We now present the main results of our paper.

\section{Results}

The distance between a vertex $x$ and a set of vertices $S$, denoted by $d(S, x)$, is the minimum distance between $x$ and a vertex of $S$. The following easy property of LexBFS holds for arbitrary graphs.

Lemma 1 Let $S_{i}$ be the numbered vertices at step $i$ of LexBFS. If $x \notin S_{i}$ and $y \notin S_{i}$ are two vertices such that $d\left(S_{i}, x\right)<d\left(S_{i}, y\right)$, then $x$ will be numbered before $y$.

Let $\sigma=\left(v_{1}, v_{2}, \ldots, v_{n}\right)$ be an ordering of the vertex set of a graph $G$. We write $a<b$ whenever in a given ordering $\sigma$ vertex $a$ has a smaller number than vertex $b$. Moreover, $\left\{a_{1}, \cdots, a_{l}\right\}<\left\{b_{1}, \cdots, b_{k}\right\}$ is an abbreviation for $a_{i}<b_{j}$ $(i=1, \cdots, l ; j=1, \cdots, k)$. An ordering of the vertex set of a graph $G$ generated by LexBFS is called a LexBFS-ordering. 
In what follows we will often use the following property (cf.[22]) :

If $a<b<c$ and $a c \in E$ and $b c \notin E$ then there exists a vertex $d$ such that $c<d, d b \in E$ and $d a \notin E$.

It is well-known that any LexBFS-ordering has property $(P 1)$ [19]. Moreover, any ordering fulfilling $(P 1)$ can be generated by LexBFS [14].

We now note that the LexBFS algorithm is guaranteed to find the diameter for arbitrary graphs, if the diameter equals 2 .

Proposition 1 Let $G$ be an arbitrary graph and let $u$ be the vertex of $G$ visited last by a LexBFS. If $\operatorname{diam}(G)=2$, then ecc $(u)=\operatorname{diam}(G)$.

Proof: Let $(x, y)$ be a diametrical pair of vertices of $G$, i.e. $d(x, y)=\operatorname{diam}(G)=$ 2 . To prove the proposition we just have to show that $u$ is not a universal vertex. Assume both vertices $x$ and $y$ are adjacent to $u$. Then, since $u<\{x, y\}$, by $(P 1)$ there exists a vertex $t>\{x, y\}$ such that $t u \notin E$, thereby proving that $u$ is not universal.

\subsection{Chordal Graphs}

We now turn our attention to chordal graphs. An ordering $\sigma$ of the vertex set of a graph $G$ is a perfect elimination ordering if $b c \in E$ for all vertices $a, b$ and $c$ with $a<\{b, c\}$ and $a b, a c \in E$. The following theorem presents the well-known characterization of chordal graphs.

Theorem 2 [27] Let $\sigma$ be a LexBFS-ordering of a graph $G$. Then $G$ is a chordal graph if and only if $\sigma$ is a perfect elimination ordering of $G$.

It is interesting to note that Maximum Cardinality Search (MCS) exhibits the same property as LexBFS with respect to perfect elimination orderings of chordal graphs [29]. For MCS, unlike LexBFS, the eccentricity of the last vertex is not guaranteed to be within any constant of the diameter of the (chordal) graph. (Consider an arbitrarily long path where a new vertex $\mathrm{x}$ is adjacent just to a midpoint of the path; it is straightforward to construct an MCS that ends at $\mathrm{x}$. )

Let $P=\left(x_{0}-x_{1}-\cdots-x_{k-1}-x_{k}\right)$ be an arbitrary path of $G$ and let $\sigma$ be an ordering of the vertex set of this graph. The path $P$ is monotonic (with respect to $\sigma$ ) if $x_{0}<x_{1}<\cdots<x_{k-1}<x_{k}$ holds whenever $x_{0}<x_{k}$, and $P$ is convex if there is an index $i(1 \leq i<k)$ such that $x_{0}<x_{1}<\cdots<x_{i-1}<$ $x_{i}>x_{i+1}>\cdots>x_{k-1}>x_{k}$. Then vertex $x_{i}$ is called the switching point of the convex path $P$.

In the remainder of this subsection we assume that $G$ is a chordal graph and $\sigma$ is a LexBFS-ordering of $G$.

By theorem 2 no induced path $P=\left(x_{0}-\cdots-x_{k}\right)$ of $G$ can contain a vertex $x_{j}(1 \leq j<k)$ with $x_{j-1}>x_{j}<x_{j+1}$. Hence, we have the following.

Lemma 2 Every induced path of $G$ is either monotonic or convex. 
Now let $P=\left(x_{0}-\cdots-x_{k}\right)$ be a shortest path of $G$ connecting $x_{0}$ and $x_{k}$. We say that $P$ is a rightmost shortest path if the sum $x_{0}+x_{1}+\cdots+x_{k}$ of the positions of $x_{0}, \cdots, x_{k}$ in $\sigma$ is largest among all shortest paths connecting $x_{0}$ and $x_{k}$.

Lemma 3 Let $P=\left(x_{0}-\cdots-x_{2 k}\right)$ be a shortest path in $G$ such that the subpath $P^{\prime}=\left(x_{i}-\cdots-x_{2 k}\right), i \geq k$, of $P$ is a rightmost shortest path connecting $x_{i}$ and $x_{2 k}$. If $x_{0}<x_{2 k}$ and $x_{k}$ is the switching point of $P$, then $x_{k+j}>x_{k-j}$ holds for each $j(i-k+1 \leq j \leq k)$.

Proof: We will show that, for each $j(i-k+1 \leq j \leq k-1)$, if $x_{k+j}<x_{k-j}$ then $x_{k+j+1}<x_{k-j-1}$ holds too. Since $x_{2 k}>x_{0}$ this will give a contradiction.

So, let $x_{k+j}<x_{k-j}$ but $x_{k+j+1}>x_{k-j-1}$. Since $P$ is a convex path and $x_{k}$ is the switching point of it we have $x_{k+j+1}<x_{k+j}$ and hence $x_{k+j+1}<x_{k-j}$. Applying ( $P 1)$ to $x_{k-j-1}<x_{k+j+1}<x_{k-j}$ we find a vertex $t>x_{k-j}$ adjacent to $x_{k+j+1}$ and not to $x_{k-j-1}$. From $x_{k+j+1}<x_{k+j}<\left\{t, x_{k+j-1}\right\}$ and theorem 2 we deduce that $t$ is adjacent to both $x_{k+j}$ and $x_{k+j-1}$. Then, a contradiction arises to $P^{\prime}$ being a rightmost shortest path, since $t>x_{k+j}$ and $t x_{k+j+1}, t x_{k+j-1} \in E$.

Lemma 4 Let $P=\left(x_{0}-\cdots-x_{k}\right)$ be a rightmost shortest path in $G$ which is convex and let $x_{i}$ be the switching point of $P$. Then $d\left(x_{0}, x_{i}\right) \geq d\left(x_{k}, x_{i}\right)$ whenever $x_{0}<x_{k}$.

Proof: We prove the assertion by induction on $k$. Note that any subpath of a rightmost shortest path is again a rightmost shortest path. For $k=2$ evidently the assertion holds. So, let $k \geq 3$. Since $P$ is convex we have $x_{k}<x_{k-1}$ and hence $x_{0}<x_{k-1}$. By the induction hypothesis, $d\left(x_{0}, x_{i}\right) \geq d\left(x_{k-1}, x_{i}\right)$. If $d\left(x_{0}, x_{i}\right) \geq d\left(x_{k-1}, x_{i}\right)+1$ then $d\left(x_{0}, x_{i}\right) \geq d\left(x_{k}, x_{i}\right)$ and we are done. So, assume that $d\left(x_{0}, x_{i}\right)=d\left(x_{k-1}, x_{i}\right)$. Since $d\left(x_{1}, x_{i}\right)<d\left(x_{k-1}, x_{i}\right)$ by the induction hypothesis we must have $x_{k-1}<x_{1}$. Moreover, from $x_{k}<x_{k-1}$ we conclude $x_{k}<x_{1}$. Applying now $(P 1)$ to $x_{0}<x_{k}<x_{1}$ we get a vertex $t>x_{1}$ adjacent to $x_{k}$ and not to $x_{0}$. From $x_{k}<x_{k-1}<\left\{t, x_{k-2}\right\}$ and theorem 2 we deduce that $t$ is adjacent to both $x_{k-1}$ and $x_{k-2}$. Then, a contradiction arises to $P$ being a rightmost shortest path, since $t>x_{k-1}$ and $t x_{k}, t x_{k-2} \in E$.

Let $u$ be the vertex of a chordal graph $G$ visited last by a LexBFS.

Lemma 5 For every two vertices $x$ and $y$ of $G$ such that $d(x, u) \leq d(y, u)$, $d(x, y) \leq d(y, u)+1$ holds. Moreover, if $d(x, y)=d(y, u)+1$ then $d(y, u)=$ $d(x, u)$ and $d(y, u)$ is even.

Proof: Assume that $d(x, y) \geq d(y, u)+1$. Consider in $G$ rightmost shortest paths $P_{x}$ and $P_{y}$, connecting vertex $u$ with vertices $x$ and $y$, respectively. Let $a$ be the common vertex of the paths $P_{x}$ and $P_{y}$ furthest from $u$. Note that, since a subpath of a rightmost shortest path is again a rightmost shortest path, paths $P_{x}$ and $P_{y}$ coincide in the part from $u$ to $a$ and do not have any other common vertices. Denote the common subpath of these paths by $P_{a}$. From $d(x, u) \leq d(y, u)$, we conclude $d(x, a) \leq d(y, a)$.

By lemma $2, P_{x}$ and $P_{y}$ are monotonic or convex. First we show that these paths cannot have a switching point on the subpath $P_{a}$. Assume by way of 
contradiction that a vertex $z$ of $P_{a}$ is the switching point of $P_{x}$ or $P_{y}$. Then by lemma 4 we obtain $d(u, z) \geq d(x, z)$, if $z$ is the switching point of $P_{x}$, or $d(u, z) \geq d(y, z)$ if $z$ is the switching point of $P_{y}$. Since $d(x, u) \leq d(y, u)$, in both cases we have $d(u, z) \geq d(x, z)$. Hence,

$$
\begin{gathered}
d(x, y) \leq d(x, a)+d(a, y) \leq d(x, z)+d(z, y) \leq \\
\leq d(u, z)+d(z, y)=d(u, y) \leq d(x, y)-1,
\end{gathered}
$$

a contradiction.

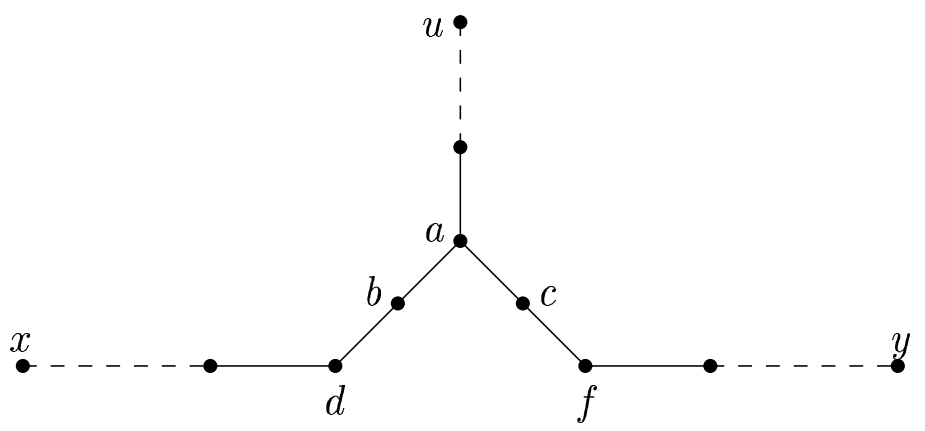

Figure 3:

Now let $b$ and $c$ be the neighbours of $a$ in the paths $P_{x}$ and $P_{y}$, respectively, which do not belong to the monotonic path $P_{a}$ (see Figure 3). Since $a<\{b, c\}$ by theorem 2 we get $b c \in E$.

Suppose $c<f$, where $f$ is the neighbour of $c$ in the path $P_{y}$ distinct from $a$. If $b>c$ then by theorem 2 vertices $b$ and $f$ will be adjacent, contradicting $P_{y}$ being rightmost. Hence, $b<c$ must hold. Now from the fact that the path $P_{x}$ is rightmost we deduce that $d c \notin E$ and $d<b$, where $d$ is the neighbour of $b$ in the path $P_{x}$ distinct from $a$. Since $a<b>d$ the path $P_{x}$ is convex and $b$ is the switching point of $P_{x}$. By lemma 4 we obtain $d(u, b) \geq d(x, b)$ and hence $d(u, c) \geq d(x, b)$. From $d(x, y) \leq d(x, b)+1+d(y, c) \leq d(u, c)+$ $d(y, c)+1=d(u, y)+1$ and our assumption that $d(x, y) \geq d(u, y)+1$ we see that $d(u, b)=d(x, b)$. Hence from $u<x$ and lemma 3 it follows that $a<d$. Now we can apply $(P 1)$ to $a<d<c$ and get a vertex $t>c$ adjacent to $d$ and not to $a$. Since $t>c>b>d$ and $c<f$ by theorem 2, vertex $t$ must be adjacent to $b, c, f$ (see Figure 4). Thus,

$$
d(x, y) \leq d(x, d)+2+d(f, y)=d(x, b)+d(y, c) \leq d(u, c)+d(y, c)=d(u, y),
$$

and a contradiction to the assumption $d(x, y) \geq d(u, y)+1$ arises.

Hence, $c>f$, and therefore $P_{y}$ is a convex path and $c$ is the switching point of $P_{y}$. Again by lemma 4 we have $d(u, c) \geq d(c, y) \geq d(b, x)$. Hence,

$$
d(x, y) \leq d(x, b)+1+d(c, y) \leq d(u, c)+1+d(c, y)=d(u, y)+1 .
$$

Since our assumption was $d(x, y) \geq d(u, y)+1$ we conclude $d(x, y)=d(u, y)+1$ and $d(u, c)=d(c, y)=d(b, x)$, i.e. $d(u, y)$ is even and $d(u, y)=d(u, x)$. 


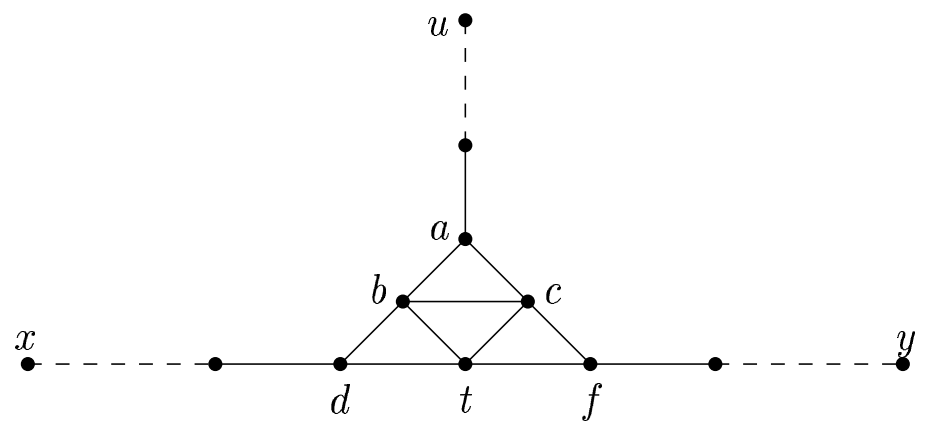

Figure 4:

Theorem 3 Let $u$ be the vertex of a chordal graph $G$ last visited by a LexBFS, and let $x, y$ be a pair of vertices such that $d(x, y)=\operatorname{diam}(G)$. If ecc $(u)<$ $\operatorname{diam}(G)$ then ecc $(u)$ is even, $d(u, x)=d(u, y)=\operatorname{ecc}(u)$ and ecc $(u)=\operatorname{diam}(G)-$ 1.

Proof: Assume, without loss of generality, that $d(u, x) \leq d(u, y)$. Then lemma 5 gives $d(x, y) \leq d(u, y)+1$. On the other hand we have $d(x, y)=\operatorname{diam}(G) \geq$ $e c c(u)+1 \geq d(u, y)+1$. Hence, $e c c(u)=d(u, y), d(x, y)=d(u, y)+1$ and $\operatorname{diam}(G)=e c c(u)+1$. Applying again lemma 5 we conclude $d(u, x)=d(u, y)=$ $e c c(u)$ and $e c c(u)$ is even.

We continue with rather surprising results concerning the parity of the diameter of the graph and the parity of the eccentricity of the vertex visited last by LexBFS.

Corollary 1 If the diameter of a chordal graph $G$ is even, then the vertex last visited by a LexBFS has eccentricity equal to diam $(G)$.

Corollary 2 If the vertex $u$ of a chordal graph $G$ last visited by a LexBFS has odd eccentricity, then ecc $(u)=\operatorname{diam}(G)$.

To prove the final result on chordal graphs we need the following auxiliary lemmas, the first two of which are well-known.

Lemma 6 For every two vertices $x, y$ of a chordal graph $G$ and every $k, 0<$ $k \leq d(x, y)$, the set $S_{k}(x, y)=\{z \in V: d(z, x)=k$ and $d(z, y)=d(x, y)-k\}$ induces a complete subgraph of $G$.

Lemma 7 If all vertices of a complete subgraph $C$ of a chordal graph $G$ have the same distance $k$ from a vertex $x$, then there is a common neighbour $z$ of all vertices of $C$ which is at distance $k-1$ from $x$.

Lemma 8 Let $G$ be a chordal graph which does not contain an induced 3-sun (see figure 5), let $w, x$ be arbitrary vertices of $G$ and let $u$ be the vertex visited last by LexBFS(w). Also let $P$ be a rightmost (with respect to LexBFS $(w)$ ) shortest path connecting $u$ with $x$ and assume that $d(u, x)=2 k$. Then the vertex $z$ of $P$ with $d(u, z)=k$ lies on a shortest path connecting vertices $w$ and $u$. 

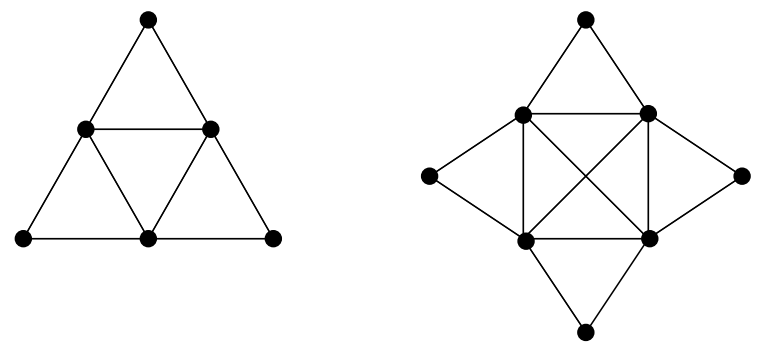

Figure 5: The 3-sun and the 4-sun.

Proof: Consider in $G$ a rightmost shortest path $P^{\prime}$ connecting vertex $u$ with $w$ and let $a$ be the common vertex of the paths $P$ and $P^{\prime}$ furthest from $u$. By lemma 2, the path $P$ is monotonic or convex (note that the path $P^{\prime}$ is monotonic since $w$ is the vertex with the largest index in $\operatorname{LexBFS}(w))$. Let $b$ and $c$ be two neighbours of $a$ in the paths $P^{\prime}$ and $P$, respectively, which are at distance $d(u, a)+1$ from $u$. (The degenerate cases where $a=x$ or $w=a$ are trivial.) We will show that $d(u, a) \geq k-1$ and if $d(u, a)=k-1$ then the vertex $c$ belongs to a shortest path connecting $w$ with $u$.

If $a>c$ then the path $P$ is convex and $a$ is the switching point of $P$. By lemma $4 d(u, a) \geq d(a, x)$. Since $d(u, x)=2 k$ we have $d(u, a) \geq k$.

Assume now that $c>a$. Then from $a<\{b, c\}$ and theorem 2 we get $b c \in E$. As before, denote by $d$ and $f$ the neighbours of $b$ and $c$, respectively, on the paths $P^{\prime}$ and $P$ distinct from $a$. We have $a<b<d$ and $a<c$. If $b<c$ then by theorem $2 d c \in E$ and hence the path $P^{\prime}$ is not rightmost, a contradiction. So, $b>c$. Analogously, since $P$ is rightmost $c>f$ and $b f \notin E$ must hold. Thus, the path $P$ is convex and $c$ is the switching point of $P$. From $d(u, x)=2 k$ and lemma 4 we obtain $d(u, c) \geq k$, i.e. $d(u, a) \geq k-1$. For the case $d(u, c)=k=d(u, a)+1$ we will show that the vertex $c$ is adjacent to $d$. Indeed, if $d(u, c)=k$ then by lemma $3 a<f$. Hence, we can apply $(P 1)$ to $a<f<b$ and get a vertex $t>b$ adjacent to $f$ and not to $a$. Since $t>b>c>f$ and $b<d$ by theorem 2 vertex $t$ must be adjacent to $c, b, d$. To avoid an induced 3 -sun we must have an edge $d c$ (note that $G$ as a chordal graph does not have any induced cycles).

Lemma 9 Let $G$ be a chordal graph which does not contain an induced 3-sun, $w, x, v$ be arbitrary vertices of $G$ and $u$ be the vertex visited last by LexBFS $(w)$. If $d(u, x)=d(u, v)=d(x, v)=2 k$, then there exists a vertex a of $G$ such that $d(u, a)=d(x, a)=d(v, a)=k$ and $d(w, a)=d(w, u)-k$.

Proof: Let $P_{x}$ and $P_{v}$ be rightmost (with respect to LexBFS $(w)$ ) shortest paths connecting $u$ with $x$ and $v$, respectively. Denote by $b$ and $c$ the midvertices of the paths $P_{x}$ and $P_{v}$, i.e. vertices with $d(x, b)=d(v, c)=k$. By lemma 8 both vertices $b$ and $c$ belong to shortest paths joining $w$ with $u$. Hence, if $c=b$ or $d(c, x)=k$ or $d(b, v)=k$ then we are done. So, assume that $c \neq b$ and $d(c, x)>k, d(b, v)>k$. Since $d(u, b)=d(u, c)=k$ and $d(w, b)=d(w, c)=d(w, u)-k$, by lemmas 6 and 7 , we get that $b c \in E$ and there are two vertices $u^{\prime}, w^{\prime}$ in $G$ such that $u^{\prime} b, u^{\prime} c, w^{\prime} b, w^{\prime} c \in E$ and $d\left(w^{\prime}, w\right)=d(w, b)-1, d\left(u^{\prime}, u\right)=d(u, b)-1$. Now consider a cycle of $G$ formed by edge $b c$ and shortest paths connecting $b$ with $x, c$ with $v$ and $v$ with $x$. 
Since $G$ is chordal, in this cycle, the middle vertex $e$ of the $(v, x)$-path must be adjacent to both $b$ and $c$. Vertices $c$ and $e$ are equidistant from $v$ as well as vertices $b$ and $e$ are equidistant from $x$. By lemma 7 there must be vertices $x^{\prime}$ and $v^{\prime}$ in $G$ such that $x^{\prime} b, x^{\prime} e, v^{\prime} e, v^{\prime} c \in E$ and $d\left(v^{\prime}, v\right)=d\left(x^{\prime}, x\right)=k-1$. Since $\{d(v, b), d(x, c)\}>k, v^{\prime} b, x^{\prime} c \notin E$. Hence, to avoid an induced 3-sun, vertex $e$ must be adjacent to both $u^{\prime}$ and $w^{\prime}$. It is easy to see now that $e$ is at distance $k$ from $u, x$ and $v$ and at distance $d(u, w)-k$ from $w$.

The main result of this subsection is the following.

Theorem 4 If $G$ is a chordal graph and if $v$, the vertex returned by algorithm 2, is not of maximum eccentricity, then $G$ contains either an induced 3-sun or an induced 4-sun (see figure 5) or one of the graphs from figure 6 as an induced subgraph.
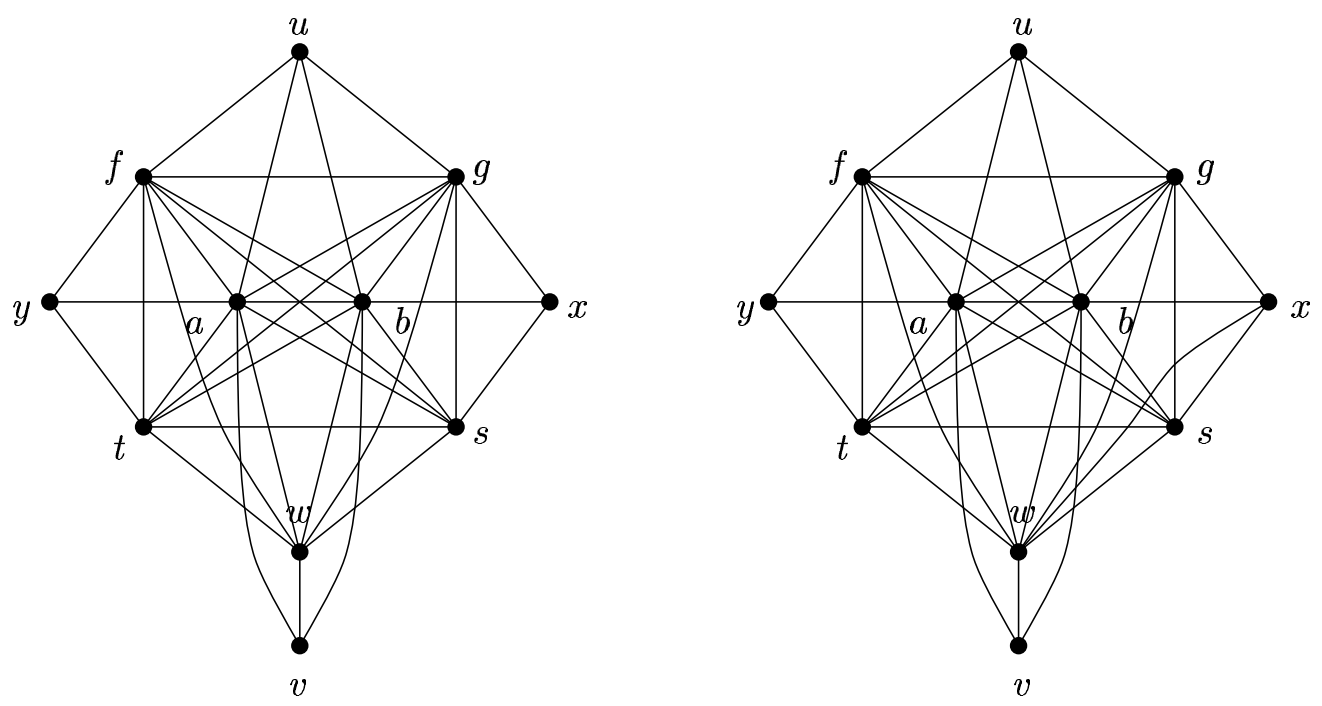

Figure 6: Strongly chordal graphs where 2 sweeps of LexBFS are not enough to find the diameter. $\operatorname{LexBFS}(w)$ and LexBFS $(u)$ orderings of the left graph are $(u, x, y, v, g, f, a, b, s, t, w)$ and $(v, x, y, s, t, w, a, b, g, f, u)$, respectively. Here we have $\operatorname{ecc}(v)=2<3=d(x, y)=\operatorname{diam}(G)$.

Proof: Let $w^{*}$ be an arbitrary vertex of $G$, let $u^{*}$ be the vertex numbered last by $\operatorname{LexBFS}\left(w^{*}\right)$ and let $v^{*}$ be the vertex numbered last by LexBFS $\left(u^{*}\right)$. We will show that if $e c c\left(v^{*}\right)<\operatorname{diam}(G)$ and $G$ contains neither induced 3-suns nor induced 4-suns then $G$ must contain one of the graphs from figure 6 as an induced subgraph.

Let $x^{*}, y^{*}$ be a diametral pair of vertices of $G$, i.e. $d\left(x^{*}, y^{*}\right)=\operatorname{diam}(G)$. Since $\operatorname{ecc}\left(v^{*}\right)<\operatorname{diam}(G)$, by theorem 3 , we have ecc $\left(u^{*}\right)=\operatorname{ecc}\left(v^{*}\right)=\operatorname{diam}(G)-$ $1, \operatorname{ecc}\left(v^{*}\right)$ is even, say ecc $\left(v^{*}\right)=2 k$, and $d\left(u^{*}, x^{*}\right)=d\left(u^{*}, y^{*}\right)=d\left(u^{*}, v^{*}\right)=$ $d\left(v^{*}, x^{*}\right)=d\left(v^{*}, y^{*}\right)=2 k=d\left(x^{*}, y^{*}\right)-1$. Applying lemma 9 to $x^{*}, u^{*}, v^{*}, w^{*}$ and to $y^{*}, u^{*}, v^{*}, w^{*}$ we will find two vertices $a$ and $b$ such that $d\left(u^{*}, a\right)=$ $d\left(y^{*}, a\right)=d\left(v^{*}, a\right)=d\left(u^{*}, b\right)=d\left(x^{*}, b\right)=d\left(v^{*}, b\right)=k$ and $d\left(w^{*}, b\right)=d\left(w^{*}, a\right)=$ 
$d\left(w^{*}, u^{*}\right)-k$. Denote $d\left(w^{*}, u^{*}\right)-k$ by $l$. By lemmas 6 and 7 vertices $a$ and $b$ are adjacent and have three common neighbours $u, v$ and $w$ such that $u, v$ are at distance $k-1$ from $u^{*}, v^{*}$, respectively, and $w$ is at distance $l-1$ from $w^{*}$ (see figure 7). We choose $v$ rightmost in $\operatorname{LexBFS}\left(w^{*}\right)$, i.e. $v$ has the largest index in LexBFS $\left(w^{*}\right)$ among all vertices which are adjacent to $a, b$ and at distance $k-1$ from $v^{*}$.

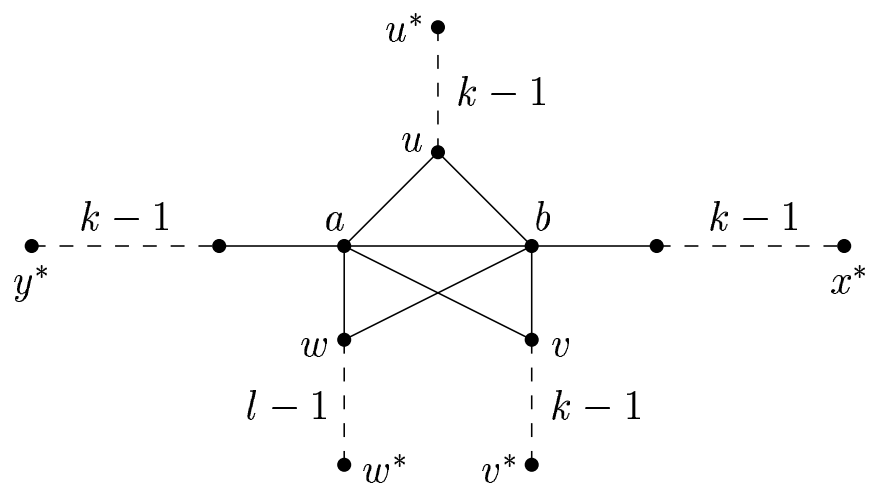

Figure 7:

Let $\sigma$ be the LexBFS $\left(w^{*}\right)$-ordering of the vertex set of $G$ with last visited vertex $u^{*}$ (note that only in the proof of claim 3 do we use the LexBFS $\left(u^{*}\right)$ ordering). From lemma 1 and distance requirements we derive $w>\{a, b\}>u$ and $u w, u v \notin E$. Without loss of generality assume that $a<b$.

Claim 1: In the LexBFS( $\left.w^{*}\right)$-ordering, vertex $u$ has a smaller index than vertex $v$.

Proof of Claim 1: If $a<v$ then we are done since $u<a$. So, assume $a>v$. Consider a rightmost shortest path $P_{v}$ connecting vertex $v$ with $v^{*}$ and an arbitrary shortest path $P_{u}$ joining $u$ with $u^{*}$. Let $u^{\prime}$ and $v^{\prime}$ be the neighbours of $u$ and $v$, respectively, on the paths $P_{u}$ and $P_{v}$. Since $\left.u<a\right\rangle v$ the shortest path $P$ formed by $P_{u}, P_{v}$ and edges $u a, a v$ is convex and $a$ is the switching point of $P$. Consequently, $u^{\prime}<u$ and $v^{\prime}<v$. Moreover, from $u^{*}<v^{*}$ and lemma 3 we get $u^{\prime}<v^{\prime}$. Now, if $v<u$ then $u^{\prime}<v^{\prime}<u$ and we can apply $(P 1)$ and find a vertex $z>u$ adjacent to $v^{\prime}$ and not to $u^{\prime}$. Since $v^{\prime}<v<z$ and $v<a<b$ by theorem $2 z$ is adjacent to $v, a, b$. This gives a contradiction to the choice of $v$ ( $z$ is adjacent to both $a$ and $b$, is at distance $k-1$ from $v^{*}$, but $z>u>v$ ).

Claim 2: There exist two adjacent vertices $t$ and $s$ in $G$ such that $d\left(x^{*}, s\right)=$ $d\left(y^{*}, t\right)=k$ and both $t$ and $s$ are adjacent to $a, b, w$ and not to $u$. Moreover, $a<s$ and $b<t$ hold in LexBFS $\left(w^{*}\right)$.

Proof of Claim 2: $\quad$ Pick the neighbour $d$ of $a$ in a rightmost shortest path connecting $a$ with $y^{*}$ and the neighbour $e$ of $b$ in a rightmost shortest path connecting $b$ with $x^{*}$. From distance requirements we have $d(e, d)=3$ and $u d, u e \notin E$. Note that $w$ may be adjacent to $d$ or $e$ but only to one of them. Since $d b \notin E$ and $a<b$ we get $d<a$. Hence, we can apply lemma 3 to a shortest path connecting $u^{*}$ with $y^{*}$ and passing through vertices $u, a, d$ and get $u<d$. Thus, $u<d<b, b u \in E$ and $d b \notin E$ hold. By property $(P 1)$ there 
must be a vertex $t>b$ adjacent to $d$ and not to $u$. Since $d<a<b<t$ and $b<w$, by theorem $2, t$ is adjacent to $a, b, w$. It cannot be adjacent to $e$ because of $d(e, d)=3$. Consequently, $e<b$ must hold yielding $u<e$ (similar as $u<d$ ). If now $a<e$ then $(P 1)$ applied to $a<e<t$ gives a vertex $p>t$ adjacent to $e$ and not to $a$. From $e<b<t<p$ and theorem 2 vertex $p$ is adjacent to $b$ and $t$. But then vertices $u, a, b, d, t$ and $p$ induce a 3 -sun, a contradiction.

Hence, $a>e$. Moreover, every vertex adjacent to both $b$ and $t$ must be adjacent to $a$ as well, otherwise we will have again an induced 3 -sun. Now we apply $(P 1)$ to $u<e<a$ and get a vertex $s>a$ adjacent to $e$ and not to $u$. From $e<\{b, s\}$ vertices $b$ and $s$ are adjacent. If $s t \notin E$ then $b>s$ holds and $(P 1)$ applied to $a<s<t$ gives a vertex $p>t$ adjacent to $s$ and not to $a$. From $s<b<t<p$ and theorem 2 vertex $p$ is adjacent to $b$ and $t$. Since $a p \notin E$ there is a 3 -sun on $u, a, b, d, t, p$. Thus, $s t \in E$ and hence $s a \in E$, otherwise there is a 3 -sun on $s, b, t, a, d, u$. From $a<\{w, s\}$, vertex $s$ is adjacent to $w$ too. Finally we note that $d\left(x^{*}, s\right)=d\left(y^{*}, t\right)$ since otherwise $d\left(x^{*}, y^{*}\right)<2 k+1$.

Claim 3: There exist two adjacent vertices $f$ and $g$ in $G$ such that $d\left(x^{*}, g\right)=$ $d\left(y^{*}, f\right)=k$ and both $f$ and $g$ are adjacent to $a, b, u$ and not to $v$.

Proof of Claim 3: This proof is similar to the proof of claim 2. Instead of the $\operatorname{LexBFS}\left(w^{*}\right)$-ordering we use the $\operatorname{LexBFS}\left(u^{*}\right)$-ordering of the vertex set of $G$ with last visited vertex $v^{*}$.

Now we have all the prerequisites to construct one of the graphs of figure 6 . Again let $\sigma$ be a LexBFS $\left(w^{*}\right)$-ordering of $G$ with last visited vertex $u^{*}$. From the discussion above we have a subgraph of $G$ presented in figure 8(a).

Since $b, g, s \in S_{k}\left(x^{*}, y^{*}\right)$, by lemmas 6 and 7 vertices $b, g, s$ are pairwise adjacent and they have a common neighbour $x$ which is at distance $k-1$ from $x^{*}$. Analogously, vertices $a, f, t$ are pairwise adjacent and have a common neighbour $y$ at distance $k-1$ from $y^{*}$. From distance requirements we have $d(x, y)=3$ and $u x, u y, u w, u v, v x, v y \notin E$. Note that $w$ may be adjacent to $x$ or $y$ but only to one of them. Recall also that $u s, u t, v f, v g \notin E$ (see claims 2 and 3). Furthermore, each vertex adjacent to both $t$ and $g$ must be adjacent to $f$ too, otherwise an induced 4-cycle or an induced 3-sun arises. Similarly, each vertex adjacent to both $f$ and $s$ must be adjacent to $g$ as well. Hence, $g t, f s \in E$. If $w f, w g \notin E$ then vertices $u, f, g, y, x, t, s, w$ induce a 4 -sun (or a 4 -cycle). So, $w$ is adjacent to $f$ and hence to $g$ (or to $g$ and hence to $f$ ). Vertex $v$ is adjacent neither to $s$ nor to $t$. Indeed, if, for example, $v s \in E$ then we get an induced 3 -sun formed by $v, s, a, g, x, u$.

Thus we have constructed a subgraph of $G$ presented in figure 8(b). Only the following additional edges are possible: $w v$ and/or either $w x$ or $w y$. If $w v \in E$ then we have a graph from figure 6 as an induced subgraph of $G$. So, assume that $w v \notin E$.

Since vertices $a, b, f, g$ are at distance $l$ (recall that $l=d\left(w^{*}, u^{*}\right)-k$ ) from $w^{*}$, while vertex $w$ is at distance $l-1$ and vertex $u$ is at distance $l+1$, from lemma $1, w>\{a, b, f, g\}>u$ must hold. We had also $a<b<t, a<s$ and $u<v$ (see claims 1 and 2). From $w>b>a, w v \notin E$ and theorem 2 we derive $v<a<b$. Assume that $f<v$. Then we can apply (P1) to $f<v<w$ and find a vertex $p>w$ adjacent to $v$ and not to $f$. Again by theorem 2, $p$ is adjacent to $a, b$ and hence to $t, s$. Since $p f \notin E$ vertices $u, f, g, y, p, t, s, x$ induce either a 4 -sun or a 3 -sun (depending on whether $p$ and $g$ are adjacent). Thus, 


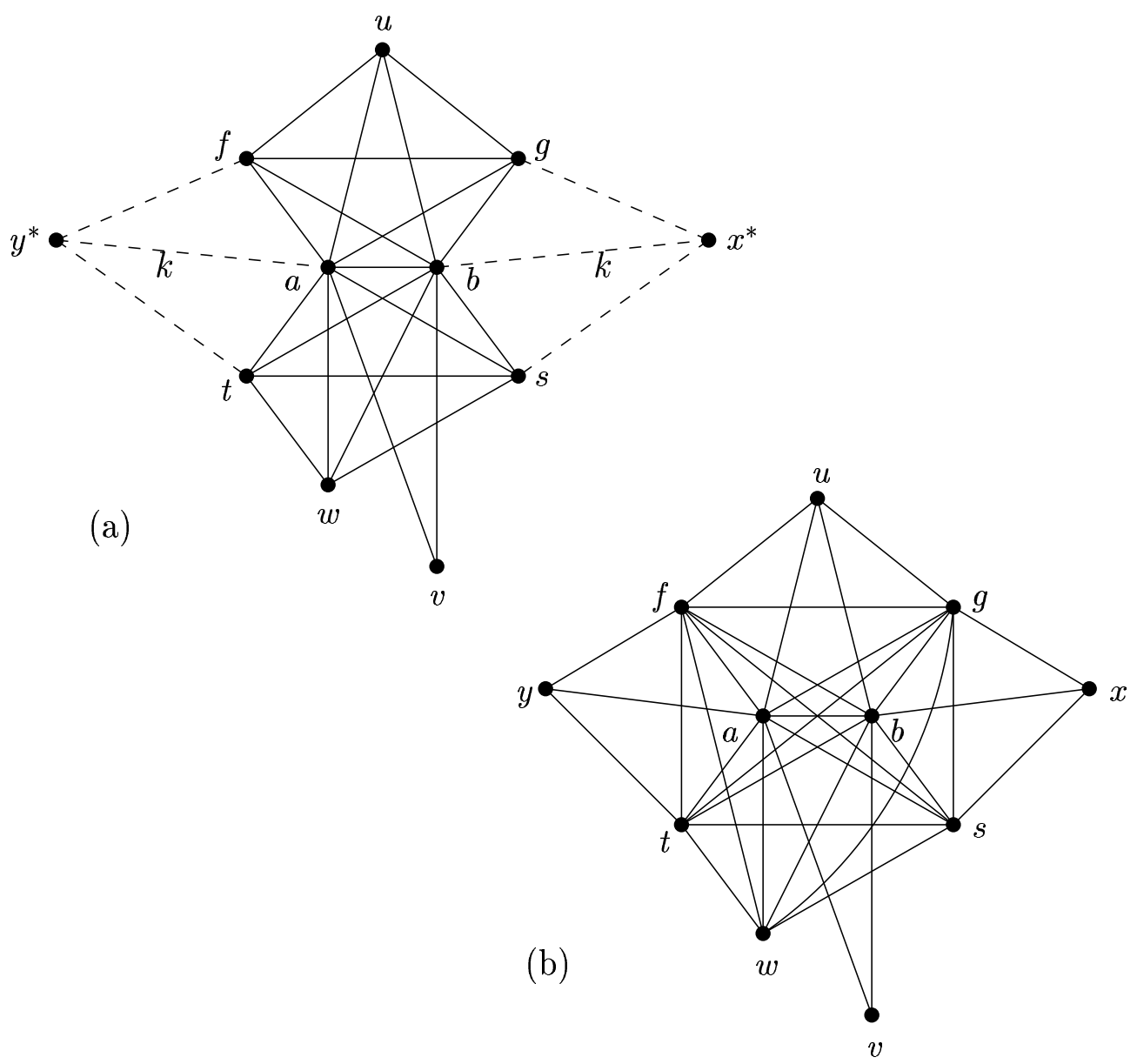

Figure 8:

we conclude $v<f$. Now we apply $(P 1)$ to $u<v<f$ and get a vertex $p>f$ adjacent to $v$ and not to $u$. As before $p$ must be adjacent to $a, b$. We will show that $p$ is adjacent to $t, s, f, g$ as well. If $p t \notin E$ then from $t>b>a$ and theorem 2 we obtain $p<a$, i.e. $p<a<b<t$ holds. Applying $(P 1)$ to $f<p<t$ we find a vertex $q>t$ adjacent to $p$ and not to $f$. Since $p<a<b<t<q$ and $a<s$, by theorem $2, q$ is adjacent to $a, b, t, s$. But then vertices $f, y, u, t, b, q$ induce a 3 -sun or a 4-cycle (if $q y \in E$ or $q u \in E$ ). Thus, vertex $p$ must be adjacent to $t$. Analogously we can show that $p s \in E$. Consequently, $p g, p f \in E$ too. Indeed, if $p g \notin E$ then vertices $p, s, a, x, g, u$ induce a 3-sun or a 4-cycle (if $p x \in E$ ), and if $p f \notin E$ then vertices $p, t, b, y, f, u$ induce a 3 -sun. Now it remains to observe that vertices $u, y, x, v, p, a, b, s, t, f$ and $g$ induce a graph from figure 6 (replace $w$ with $p$ in those pictures).

Recall that a graph $G$ is a comparability graph if one can assign directions to edges of $G$ so that the resulting digraph $G^{\prime}$ is transitive; that is, whenever $(x, y)$ and $(y, z)$ are edges of $G^{\prime}$ then $(x, z)$ is also an edge of $G^{\prime}$ [19]. Since none of the graphs from figure 5 and figure 6 is a directed path graph as well as a 
comparability graph, this theorem immediately yields the following corollary.

Corollary 3 Algorithm 2 finds a vertex of maximum eccentricity for directed path graphs and for chordal comparability graphs.

Unfortunately, this result cannot be extended to the strongly chordal graphs since for each of the graphs from figure 6, 2 sweeps of LexBFS are not enough to find the diameter. Futhermore, in figure 9 we present a strongly chordal graph for which 3 sweeps of LexBFS are also insufficient.

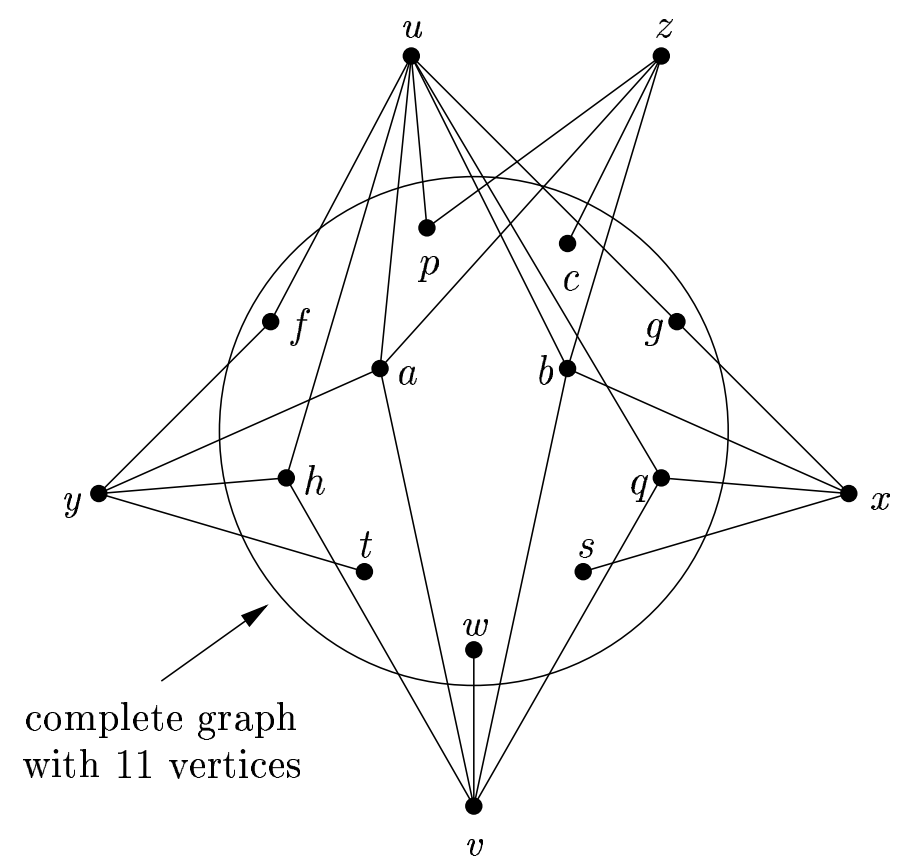

Figure 9: A strongly chordal graph where 3 sweeps of LexBFS are not enough to find the diameter. $\operatorname{LexBFS}(w), \operatorname{LexBFS}(u)$ and $\operatorname{LexBFS}(v)$ orderings are $(u, z, y, x, v, \cdots, c, t, s, w),(v, x, y, z, \cdots, g, f, p, u)$ and $(z, u, x, y, \cdots, q, h, v)$, respectively. Here we have ecc $(z)=2<3=d(x, y)=\operatorname{diam}(G)$.

\subsection{AT-free Graphs}

We now turn our attention to AT-free graphs and start by recalling some known results. A pair of vertices $(x, y)$ is said to be a dominating pair if for every $x, y$ path $P$ and every vertex $z \in V, N(z) \cap P \neq \emptyset$. If $N(z) \cap P=\emptyset$, we say that $P$ misses $z$. For vertices $u, v$ and $x$, we say that $u$ and $v$ are unrelated with respect to $x$ if there is a $v, x$ path that misses $u$ and a $u, x$ path that misses $v$. As an example of the remarkable similarity exhibited by LexBFS on chordal and AT-free graphs, we note the following characterization of AT-free graphs, a characterization that is analogous to theorem 2 for chordal graphs.

Theorem 5 [8] Let $\sigma$ be a LexBFS ordering of a connected graph $G$. If $G$ is AT-free, then for all vertices $a, b$ and $c$ with $a<\{b, c\}, b$ and $c$ are NOT unrelated with respect to a. 
In [9], it was shown that every connected AT-free graph has a dominating pair; in [8] this was strengthened to show that Algorithm 2 can be used to find such a dominating pair.

Theorem 6 [8] Let $G$ be a connected AT-free graph and let vertices $u$ and $v$ be as identified in Algorithm 2. Then $u$ and $v$ are a dominating pair of $G$.

Furthermore, as the following lemma shows, for sufficiently high diameter, the set of dominating pairs of an AT-free graph can be expressed as the Cartessian product of two distinct sets. (These sets can be found in linear time [8].)

Lemma 10 [9] If $G$ is a connected AT-free graph with diam $(G)>3$ then there exists disjoint vertex sets $X, Y$ such that $(x, y)$ is a dominating pair of $G$ iff $x \in X$ and $y \in Y$.

The fact that this lemma does not hold for $\operatorname{diam}(G)=3$ is illustrated by the graph in figure 10 .

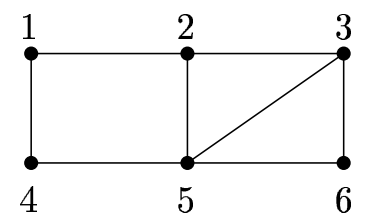

Figure 10: The dominating pairs are $(1,3),(1,6),(4,3),(4,6),(1,5),(2,5)$ and $(4,5)$. There are no disjoint sets whose cartesian product defines all dominating pairs.

A weaker version does however hold for AT-free graphs of diameter larger than or equal to 3 .

Lemma 11 Let $G$ be a connected $A T$-free graph with diam $(G) \geq 3$ and let $V_{1}$ be the set of vertices that are the last vertices of some LexBFS. Then there exists a partition of $V_{1}$ into non-empty sets $X$ and $Y$ such that $(x, y)$ is a dominating pair if $x \in X$ and $y \in Y$.

Proof: If diam $(G)>3$, then lemma 10 applies; $X$ (resp. $Y$ ) is the intersection of $V_{1}$ with the $X$ (resp. $Y$ ) identified in lemma 10 . Thus we only need to consider the case where $\operatorname{diam}(G)=3$.

Let $z$ be a vertex of maximum eccentricity and let $x$ be a vertex visited last by a LexBFS from $z$ (i.e. $x \in V_{1}$ ). We now assume that $y$ is a vertex visited last by a LexBFS from $x$. Denoting the vertices of distance $i$ from $x$ by $N^{i}(x)$ we let $X^{\prime}=V_{1} \cap N^{3}(x)$ and $Y^{\prime}=V_{1} \cap N^{3}(y)$.

Set $V_{1}^{\prime}=V_{1} \backslash\left(X^{\prime} \cup Y^{\prime}\right)$ and let $X^{\prime \prime}=V_{1}^{\prime} \cap N^{2}(x)$ and $Y^{\prime \prime}=V_{1}^{\prime} \cap N^{2}(y)$. Finally we let $X=X^{\prime} \cup X^{\prime \prime}$ and $Y=Y^{\prime} \cup Y^{\prime \prime}$. We now prove that $X$ and $Y$ satisfy the statement of the lemma.

Claim 4: $X \cap Y=\phi$

Proof of Claim 4: First we show $X^{\prime} \cap Y^{\prime}=\phi$. Suppose to the contrary that $w \in X^{\prime} \cap Y^{\prime}$ and let paths $x-a-b-w$ and $y-c-d-w$ be arbitrary $x, w$ and $y, w$ 
shortest paths. Trivially, the only possible intersection between these two paths is if $b=d$. Regardless of the intersection, $x c \notin E$ and $y a \notin E$ since otherwise $d(x, y)=2$. If $b=d, x$ and $y$ are unrelated with respect to $w$ contradicting theorem 5. If $b \neq d, x d \notin E$ (else $d(x, w)=2$ ) and $y b \notin E$, again showing that $x$ and $y$ are unrelated with respect to $w$.

$X^{\prime \prime} \cap Y^{\prime \prime}=\phi$ since otherwise the two paths $x-a-w$ and $y-b-w$, where $w \in X^{\prime \prime} \cap Y^{\prime \prime}$, must have $x b \notin E$, ya $\notin E$ and thus $x$ and $y$ would be unrelated with respect to $w$.

Note that by definition $X^{\prime} \cap Y^{\prime \prime}=X^{\prime \prime} \cap Y^{\prime}=\phi$.

Claim 5: $X \cup Y=V_{1}$

Proof of Claim 5: If $w \in V_{1} \backslash(X \cup Y)$, then $\operatorname{since} \operatorname{diam}(G)=3, w \in\{x\} \cup N^{1}(x)$ and $w \in\{y\} \cup N^{1}(y)$ which implies that $d(x, y) \leq 2$ contradicting $d(x, y)=3$.

Before proceeding to show that every element of $X$ forms a dominating pair with every element of $Y$, we note the following:

Let $u$ be an arbitrary element of $X$ and $v$ an arbitrary element of $Y$. Since $u \in X, d(x, u)=2$ or 3 . If $d(x, u)=3, d(y, u) \leq 2$ (by claim 4 ). If $d(x, u)=$ $2, d(y, u) \leq 1$ (if $d(y, u)=3, u \in Y$ contradicting claim $4 ; d(y, u) \neq 2$ since $\left.X^{\prime \prime} \cap Y^{\prime \prime}=\phi\right)$. Similarly if $d(y, v)=3, d(x, v) \leq 2$ and if $d(y, v)=2, d(x, v) \leq 1$.

Claim 6: For all $u \in X, v \in Y, u, v$ is a dominating pair

Proof of Claim 6: Suppose to the contrary, that there is a pair of vertices $u, v$ and an induced path $P$ joining them that misses vertex $t$. We now do a case by case analysis based on the distance from $x$ to $P$ and from $y$ to $P$. We let $d(x, P)$ denote the length of a shortest path from $x$ to $P$. Thus $d(x, P)=0$ means $x$ is on $P ; d(x, P)=1$ means $x$ has a neighbour on $P$ and $d(x, P)>1$ means $x$ misses $P$ (i.e. $x$ plays the role of $t$ ).

Case 1: $d(x, P)=0$ and $d(y, P)=0$ (i.e. both $x, y$ are on $P$ )

This is clearly impossible since the subpath of $P$ between $x$ and $y$ misses $t$, contradicting $x, y$ being a dominating pair.

Case 2: $d(x, P)=0$ and $d(y, P)=1$ (i.e. one vertex (without loss of generality $x$ ) is on $P$, the other is adjacent to $P$ )

First we note (and this also applies to case 3 below) that $x$ must be one of the endpoints of $P$ or adjacent to one of the endpoints. Otherwise $u$ and $v$ are unrelated with respect to $x$. Since $d(x, u)=2$ or 3 , either $x v \in E$ or $x=v$. Let $p$ be the neighbour of $x$ on $P$ in the direction towards $u$. Since $d(x, u) \geq 2, u \neq p$.

Since $d(y, P)=1$, there is a vertex $q \in P$ such that $y q \in E$. Clearly such a vertex $q$ is between $u$ and $p$ but cannot be $p$ (otherwise $d(x, y)=2$ ). The $x, y$ path $x \sim(P) \sim q-y$ (this notation indicates the subpath of $P$ from $x$ to $q$ together with the edge $q y$ ) shows that $t y \in E$ (otherwise $x, y$ is not a dominating pair). Now let $q$ be the neighbour of $y$ on $P$ that is closest to $u$. The paths $u \sim(P) \sim q-y-t$ and $u \sim(P) \sim v$ show that $v$ and $t$ are unrelated with respect to $u$. 
Case 3: $d(x, P)=0$ and $d(y, P)>1$ (i.e. one vertex (without loss of generality $x$ ) is on $P$, the other is not adjacent to $P$ )

Note that $y$ can now play the role of $t$. As in case 2 we let $p$ denote the neighbour of $x$ on $P$ and see that $p y \notin E$ (otherwise $d(x, y)=2$ ). Since $d(y, u) \leq 2$ and $d(y, P)>1$ we see that $d(y, u)=2$ and let $u-\alpha-y$ denote such a path $(\alpha \notin P)$.

We immediately see that $x \alpha \notin E$ (otherwise $d(x, y)=2$ ) and thus paths $u-\alpha-y$ and $u \sim(P) \sim x$ show that $x, y$ are unrelated with respect to $u$.

Case 4: $d(x, P)=1$ and $d(y, P)=1$ (i.e. neither $x$ nor $y$ is on $P$ but each intercepts $P$ )

The $x, y$ path that has all internal vertices on $P$ shows that $x t \in E$ or $y t \in E$ but not both. Without loss of generality assume $x t \in E$. If $u v \in E$, then $y u, x v \in E$. Now $y, t$ are unrelated with respect to $v$. Thus $u v \notin E$. Let $x^{\prime}$ be the neighbour of $x$ (on $P$ ) that is closest to $v$ and consider the paths:

$v \sim(P) \sim u$ and $v \sim(P) \sim x^{\prime}-x-t$ (where possibly $v=x^{\prime}$ ). Since $x u \notin E, t$ and $u$ are unrelated with respect to $v$ unless $x^{\prime}$ is adjacent to $u$. Thus $d(x, u)=2$ implying that $d(y, u) \leq 1$. Since $y$ is not on $P, d(y, u)=1$. Also $x^{\prime} \neq v$ since $u v \notin E$.

Now the paths $v \sim(P) \sim u-y$ and $v \sim(P) \sim x^{\prime}-x-t$ show that $y$ and $t$ are unrelated with respect to $v$ unless $y$ is adjacent to some vertex on the $v, x^{\prime}$ subpath of $P$. Let $y^{\prime}$ be the neighbour of $y$ closest to $v ; y^{\prime} \neq v$ since $d(y, v) \geq 2$. The existence of $y^{\prime}$ shows that $x^{\prime} v \notin E$. Now the path $y-u-x^{\prime}-x$ misses $v$ contradicting $x, y$ being a dominating pair. Note $x v \notin E$ since $x^{\prime}$ is the neighbour of $x$ that is closest to $v$.

Case 5: $d(x, P)=1$ and $d(y, P)>1$ (i.e. $x$ intercepts $P$ but $y$ misses $P$; note $y$ now plays the role of $t$ )

Since $d(y, P)>1$ and $d(y, u) \leq 2, d(y, u)=2$ by the path $u-\alpha-y$ where $\alpha \notin P$. Now $x y, y x^{\prime}, x \alpha \notin E$ and also $u x \notin E$. But paths $u-\alpha-y$ and $u \sim(P) \sim x^{\prime}-x$ show that $x$ and $y$ are unrelated with respect to $u$.

Case 6: $d(x, P)>1$ and $d(y, P)>1$ (i.e. both $x$ and $y$ miss $P$ )

Now $d(y, u)=2$ by the path $u-\alpha-y$ and $d(x, v)=2$ by the path $v-\beta-x$ where $\alpha, \beta \notin P(\alpha \neq \beta)$. Again $y x, y \beta, x \alpha \notin E$. But path $u-\alpha-y$ and the path induced on $u \sim(P) \sim v-\beta-x$ show that $x$ and $y$ are unrelated with respect to $u$.

This completes the proof of the lemma.

For the graph shown in figure $10, X=\{6\}$ and $Y=\{1,4\}$. The next proposition presents further facts about the structure of the AT-free graphs.

Proposition 2 Let $G$ be an AT-free graph with $\operatorname{diam}(G)=k>2$. If ecc $(v)=$ $k-1$, where $v$ is the vertex returned by algorithm 2, and $u^{\prime}, v^{\prime}$ achieve the diameter where $d\left(u, u^{\prime}\right) \leq d\left(u, v^{\prime}\right)$ then :

1. $d(u, v)=d\left(u, v^{\prime}\right)=d\left(u^{\prime}, v\right)=k-1$, 
2. $u u^{\prime} \in E$ and $v v^{\prime} \in E$.

\section{Proof:}

1. By lemma 11, $u, v$ is a dominating pair. Thus each of $u^{\prime}, v^{\prime}$ is adjacent to at least one vertex of $Q$, an arbitrary shortest $u, v$ path. Let $a$ be the furthest vertex from $u$ (on $Q$ ) that is adjacent to $u^{\prime}$ and let $b$ be the furthest vertex from $v$ (on $Q$ ) that is adjacent to $v^{\prime}$. By theorem 1, it is clear that $\operatorname{ecc}(u)=k-1$ and $d(u, v)=k-1$. Since $d\left(u^{\prime}, v^{\prime}\right)=k$, either $a=u$ or $b=v$ (or both). Without loss of generality assume $v=b$. Now $d\left(u^{\prime}, v\right)=k-1$ since otherwise $d\left(u^{\prime}, v^{\prime}\right)<k$. If $u^{\prime} u \in E$ then the same argument shows that $d\left(u, v^{\prime}\right)=k-1$. Thus we may assume that $u u^{\prime} \notin E$ and $a$ is the neighbour of $u$ on $Q$. Suppose $d\left(u, v^{\prime}\right)<k-1$ as witnessed by path $R$. Now $u^{\prime}$ must be adjacent to some vertex of $R$ since otherwise the $u, v$ path consisting of $R$ plus the edge $v^{\prime} v$ misses $u^{\prime}$ contradicting $u, v$ being a dominating pair. But now, $d\left(u^{\prime}, v^{\prime}\right)<k$.

2. This follows immediately from the preceeding argument.

Although even the 2-sweep LexBFS algorithm does not guarantee a maximum eccentricity vertex for AT-free graphs (as well as for chordal graphs), the previous proposition shows that such a vertex is in the last BFS layer from $v$, the vertex returned by algorithm 2 .

Before presenting the final result on AT-free graphs, we introduce the notion of an $h$-ladder and an $h^{-}$-ladder.

Definition 1 An h-ladder consists of a chain of $h$ 4-cycles where the 4-cycles are attached as shown in figure 11. In an $h$-*ladder the first 4-cycle has a diagonal.
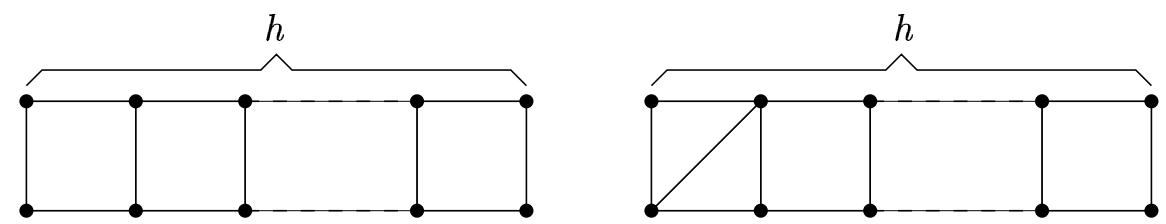

Figure 11: The h-ladder and the h-*ladder.

Theorem 7 If $G$ is an AT-free graph with $\operatorname{diam}(G)=k \geq 3$ and ecc $(v)=k-1$, where $v$ is the vertex returned by algorithm 2 , then $G$ contains an induced $(k-1)$ ladder or an induced $(k-1)-{ }^{*} l a d d e r$.

Proof: Let $u^{\prime}, v^{\prime}$ be a diametrical dominating pair such that $u, u^{\prime} \in X, v, v^{\prime} \in Y$. Clearly such $u^{\prime}, v^{\prime}$ exist (i.e. $u^{\prime}, v^{\prime} \in V_{1}$ ). Furthermore let $P$ be an arbitrary rightmost shortest $u^{\prime}, v$ path $u^{\prime}=u_{0}-u_{1}-\cdots-u_{k-1}=v$. Throughout the proof $a<b$ indicates that vertex $a$ has a smaller number than vertex $b$ in the last LexBFS of Algorithm 2. 
$L_{i}(0 \leq i \leq k-1)$ will denote the BFS layers of $G$ with respect to $u$ (i.e. $L_{0}=\{u\} ; v \in L_{k-1}$ and it has been shown in proposition 2 that $u^{\prime} \in L_{1}, v^{\prime} \in$ $\left.L_{k-1}\right)$. The following facts follow immediately:

Fact 1: For all $0 \leq i<k-1$, no neighbour of $u_{i}$ may be of distance $\leq k-i-2$ from $v^{\prime}$ (otherwise $d\left(u^{\prime}, v^{\prime}\right)<k$.)

Fact 2: For exactly one $l, 1 \leq l \leq k-1, L_{l}$ contains 2 vertices from $P$ namely $u_{l-1}, u_{l}$. All other layers contain exactly one vertex from $P$. (Pigeonhole principle and distance properties.) If $L_{l}$ contains $u_{l-1}, u_{l}$ we say that $P$ "jogs" in $L_{l}$.

Claim 7: If $P$ has its jog in $L_{l}, 1 \leq l \leq k-1$, then there is an induced $(k-l-1)$ - ladder on $\left\{u_{l}, \cdots, u_{k-1}=v\right\} \cup\left\{\alpha_{l}, \cdots, \alpha_{k-1}=v^{\prime}\right\}$ where $\alpha_{j} \in L_{j}$ for all $j$, $l \leq j \leq k-1$ and $\alpha_{j}>u_{j}$.

Proof of Claim 7: By induction on $j: k-1, \cdots, l$. If $j=k-1, \alpha_{k-1}=v^{\prime}, v^{\prime} v \in$ $E$ (i.e. we have a $[k-(k-1)-1=0]$-ladder), $v^{\prime}>v$ and $v^{\prime} \in L_{k-1}$.

Now assume the claim is true for $j>l$ and show it's true for $j-1$. $u_{j-1} \in L_{j-1}$ and thus $u_{j-1}>\alpha_{j}$. By fact $1, u_{j-1} \alpha_{j} \notin E$. Since $\alpha_{j}>u_{j}$, by $(P 1)$ there exists $\alpha_{j-1}>u_{j-1}$ such that $\alpha_{j-1} \alpha_{j} \in E, \alpha_{j-i} u_{j} \notin E$. By lemma $11, P$ is a dominating path and thus must dominate $\alpha_{j-1}$. By fact 1 , $u_{j-2} \alpha_{j-1} \notin E$ and thus $\alpha_{j-1} u_{j-1} \in E$, thereby extending the ladder.

As an immediate corollary of claim 7 , we see that if $l=1$ then $u$ is adjacent to $u_{0}\left(=u^{\prime}\right), u_{1}$ and $\alpha_{1}$, thereby resulting in a $(k-1)$-*ladder.

Henceforth we assume that $l>1$. We now show that there is only one possible ordering of $u_{l}, u_{l-1}, \alpha_{l}$.

Claim 8: $u_{l-1}>\alpha_{l}>u_{l}$

Proof of Claim 8: By claim 7, $\alpha_{l}>u_{l}$. If the stated order is not present then $\alpha_{l}>u_{l-1}$. Since $l>1, u_{l-2}$ exists, in $L_{l-1}$. By fact $1, u_{l-2} \alpha_{l} \notin E$ and thus by $(P 1)$ there exists $\alpha_{l-1}>u_{l-2}$ such that $\alpha_{l-1} u_{l-1} \notin E, \alpha_{l-1} \alpha_{l} \in E$. By fact 1 , $\alpha_{l-1} u_{l-2} \notin E$. Now consider any direct $u, \alpha_{l-1}$ path and append to it the path $\alpha_{l-1}-\alpha_{l}-\ldots-v^{\prime}$. This path (from $u$ to $v^{\prime}$ ) misses $u_{l-1}$ contradicting lemma 11.

Since $\alpha_{l}>u_{l}$, there is $\alpha_{l-1}$ (with as large a number in $\sigma$ as possible) such that $\alpha_{l-1}>u_{l-1}, \alpha_{l-1} \alpha_{l} \in E, \alpha_{l-1} u_{l} \notin E$. Furthermore, by fact $1, \alpha_{l-1} u_{l-2} \notin E$. Suppose $\alpha_{l-1} u_{l-1} \notin E$. If $\alpha_{l-1} \in L_{l-1}$, then $P$ misses $\alpha_{l-1}$ (note in this case, $\alpha_{l-1} u_{l+1} \notin E$ since $u_{l+1} \in L_{l+1}$ ). Thus $\alpha_{l-1} \in L_{l}$ and $u_{l-2}>\alpha_{l-1}$. Thus there exists $\alpha_{l-2}>u_{l-2}$ such that $\alpha_{l-2} \alpha_{l-1} \in E, \alpha_{l-2} u_{l-1} \notin E$. But now a direct path from $u$ to $\alpha_{l-2}$ concatenated with the path $\alpha_{l-2}-\alpha_{l-1}-\cdots-v^{\prime}$ yields a path from $u$ to $v^{\prime}$ that misses $u_{l-1}$, contradicting lemma 11. Thus, $\alpha_{l-1} u_{l-1} \in E$. Furthermore $\alpha_{l-1} u_{l+1} \notin E$ since otherwise the path $P^{\prime}$ formed from $P$ by replacing $u_{l}$ with $\alpha_{l-1}$ would contradict $P$ being rightmost. We now examine the possible relative orders of $u_{l-2}$ and $\alpha_{l-1}$.

Case 1: $\alpha_{l-1}>u_{l-2}$ (i.e. $\alpha_{l-1} \in L_{l-1}$ ) 
If $l=2, u$ is adjacent to $\alpha_{l-1}$ and $u_{l-2}$ and we have an induced (k-1)-ladder. If $l>2, u_{l-3}$ exists (in $L_{l-2}$ ) with $u_{l-3} u_{l-2} \in E, u_{l-3} \alpha_{l-1} \notin E$ (fact 1 ). Since $\alpha_{l-1}>u_{l-2}$, there exists $\alpha_{l-2}>u_{l-3}$ such that $\alpha_{l-2} \alpha_{l-1} \in E, \alpha_{l-2} u_{l-2} \notin E$. But now a direct $u, \alpha_{l-2}$ path concatenated with the path $\alpha_{l-2}-\alpha_{l-1}-\cdots-v^{\prime}$ yields a path from $u$ to $v^{\prime}$ that misses $u_{l-2}$, contradicting lemma 11 .

Case 2: $u_{l-2}>\alpha_{l-1}$

We differentiate on whether $\alpha_{l-1} \in L_{l}$ or $L_{l-1}$.

Case 2.1: $\alpha_{l-1} \in L_{l}$

Since $\alpha_{l-1}>u_{l-1}$, there is $\alpha_{l-2}>u_{l-2}\left(\alpha_{l-2} \in L_{l-1}\right)$ such that $\alpha_{l-2} \alpha_{l-1} \in$ $E$, and $\alpha_{l-2} u_{l-1} \notin E$. First we see that $\alpha_{l-2} \alpha_{l} \notin E$ since otherwise a direct path from $u$ to $\alpha_{l-2}$ concatenated with the path $\alpha_{l-2}-\alpha_{l}-\cdots-v^{\prime}$ yields a path from $u$ to $v^{\prime}$ that misses $u_{l-1}$, contradicting lemma 11. Secondly $\alpha_{l-2} u_{l} \notin E$ since otherwise there would exist vertex $\beta>\alpha_{l-2}$ such that $\beta \alpha_{l} \in E, \beta u_{l} \notin E$ but this would contradict $\alpha_{l-1}$ having as large a number in $\sigma$ as possible. Now we see that $\alpha_{l-2} u_{l-2} \in E$. Otherwise, if $l=2, P$ misses $\alpha_{l-2}$. If $l>2$, there exists $\alpha_{l-3}>u_{l-3}$ such that $\alpha_{l-3} \alpha_{l-2} \in E, \alpha_{l-3} u_{l-2} \notin E$ but now a direct path from $u$ to $\alpha_{l-3}$ concatenated with the path $\alpha_{l-3}-\alpha_{l-2}-\cdots-v^{\prime}$ yields a path from $u$ to $v^{\prime}$ that misses $u_{l-2}$, contradicting lemma 11 .

If $l=2, u^{\prime}=u_{l-2}$ and there is a $(k-1)$-ladder on $P \cup\{\alpha\} \cup v^{\prime}$. If $l>2$, a straightforward induction argument shows that for all $i, 1 \leq i \leq l-1, L_{l-i}$ contains vertices $\alpha_{l-i-1}>u_{l-i-1}$ where $\alpha_{l-i-1} \alpha_{l-i} \in E, \alpha_{l-i-1} u_{l-i-1} \in E$, $\alpha_{l-i} u_{l-i-1} \notin E$ and $\alpha_{l-i-1} u_{l-i} \notin E$. When $i=l-1, u_{l-i-1}=u^{\prime}$ and we have an induced $(k-1)$-ladder on $P \cup\{\alpha\} \cup v^{\prime}$.

Case 2.2: $\alpha_{l-1} \in L_{l-1}$.

If $l=2$ (i.e. $u_{l-2}=u^{\prime}$ ), then there is a $(k-1)$ )-ladder on $P \cup u \cup\{\alpha\} \cup v^{\prime}$.

If $l>2$, let $\alpha_{l-2}$ be the neighbour of $\alpha_{l-1}$ in $L_{l-2}$ with the largest number. Since $u_{l-1} \in L_{l}, \alpha_{l-2} u_{l-1} \notin E . \alpha_{l-2} u_{l-2} \in E$ since otherwise $P$ would miss $\alpha_{l-2}$. Now consider $u_{l-3}\left(\in L_{l-2}\right)$. If $l=3, u_{l-3}=u^{\prime}$ and we have a $(k-1)$ ladder on $P \cup u \cup\{\alpha\} \cup v^{\prime}$. If $l>3$ we now show that $u_{l-3}>\alpha_{l-2}$. Assume to the contrary. Since $l>3, u_{l-4}$ exists (in $L_{l-3}$ ). By fact $1, u_{l-4} \alpha_{l-2} \notin E . \alpha_{l-2}>$ $u_{l-3}$ implies there exists $\alpha_{l-3}>u_{l-4}$ such that $\alpha_{l-3} \alpha_{l-2} \in E, \alpha_{l-3} u_{l-3} \notin E$ and $u_{l-4} \alpha_{l-3} \notin E$ (by fact 1 ). Thus $P$ misses $\alpha_{l-3}$.

Now a straightforward induction argument shows that for all $i, 2 \leq i \leq$ $l-1, L_{l-i}$ contains vertices $u_{l-i-1}>\alpha_{l-i}$ where $\alpha_{l-i} \alpha_{l-i+1} \in E, \alpha_{l-i} u_{l-i} \in E$ (otherwise $P$ misses $\alpha_{l-i}$ ), $\alpha_{l-i} u_{l-i-1} \notin E$ (fact 1). When $i=l-1, u_{l-i-1}=u^{\prime}$ and we have a $(k-1)$-ladder on $P \cup u \cup\{\alpha\} \cup v^{\prime}$.

This completes the proof of the theorem.

Note that this theorem considerably strengthens the following result by Dragan [12]. An HHD-free graph does not contain an induced house (complement of $P_{5}$ ) or an induced hole (an odd cycle of length at least 5) or an induced domino (a 2-ladder). 
Theorem 8 [12] If $G$ is an HHD-free, AT-free graph, then the vertex visited last by a LexBFS has maximum eccentricity.

Similarly considering $K_{1,3}$ induced subgraphs we have:

Corollary 4 If $G$ is an AT-free graph with no $K_{1,3}$ then the vertex returned by algorithm 2 has maximum eccentricity.

\section{Concluding Remarks}

First of all, the reader should note a kind of duality in the results when algorithm 2 finds a vertex whose eccentricity is not maximum. For chordal graphs, each of the forbidden subgraphs has an AT. For AT-free graphs, the h-ladder and the h-*ladder are built with 4-cycles, the smallest non-chordal graph.

Having seen the power of the 2-sweep LexBFS algorithm, it is natural to ask whether significant improvements can be achieved by performing $c$ sweeps for some $c>2$. In particular, can we find a vertex of maximum eccentricity, although in light of the results of section 2, this is highly unlikely for $c$ a constant? As shown by the graphs in figure 12, for no $c$, is the $c$-sweep algorithm guaranteed to find a vertex of maximum eccentricity. The first graph is chordal, the second AT-free. In both graphs any LexBFS starting at $u$ must end at $v$ and vice versa. Thus if the initial choice of vertex is either $u$ or $v$, a multi-sweep LexBFS algorithm will forever alternate between $u$ and $v$, thereby missing $x$ and $y$, the two vertices of maximum eccentricity.
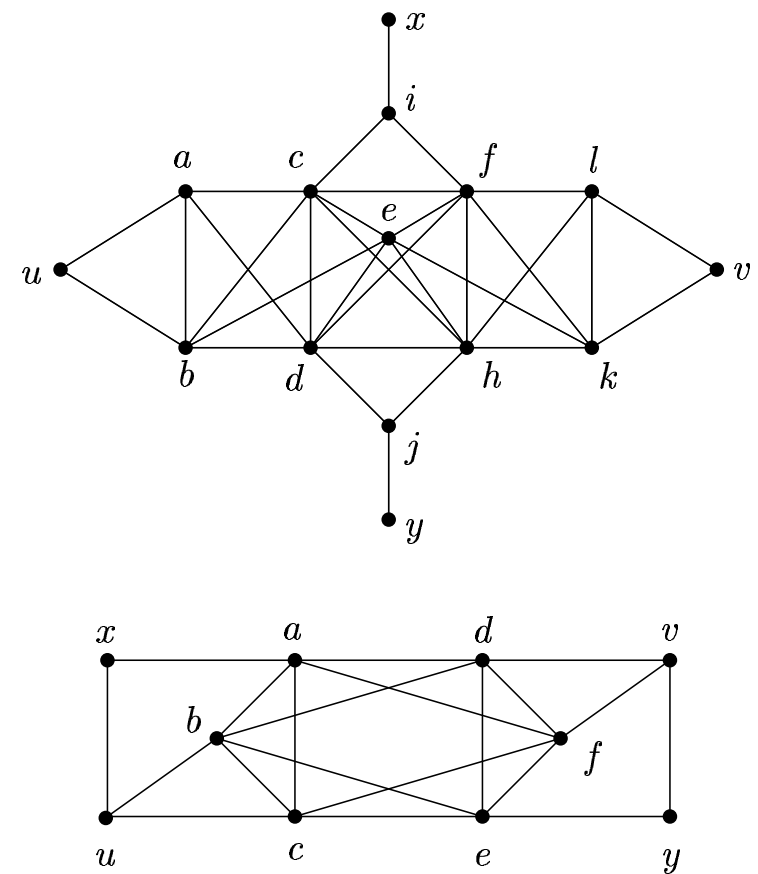

Figure 12: A chordal graph and an AT-free graph where an infinite number of LexBFS sweeps never end at a maximum eccentricity vertex. 


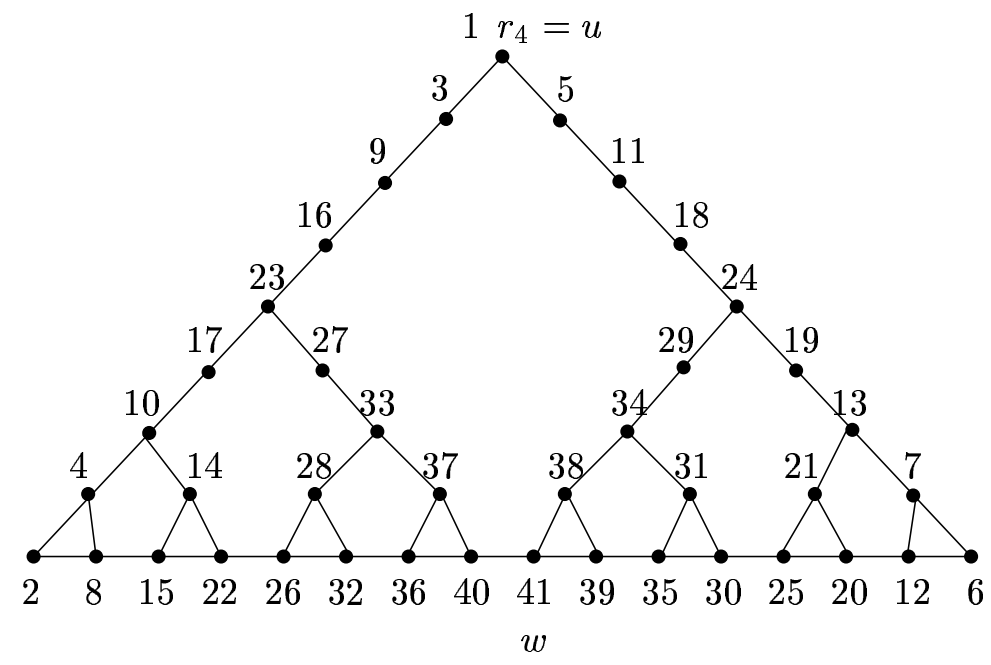

Figure 13: $G_{4}$ together with the first LexBFS.

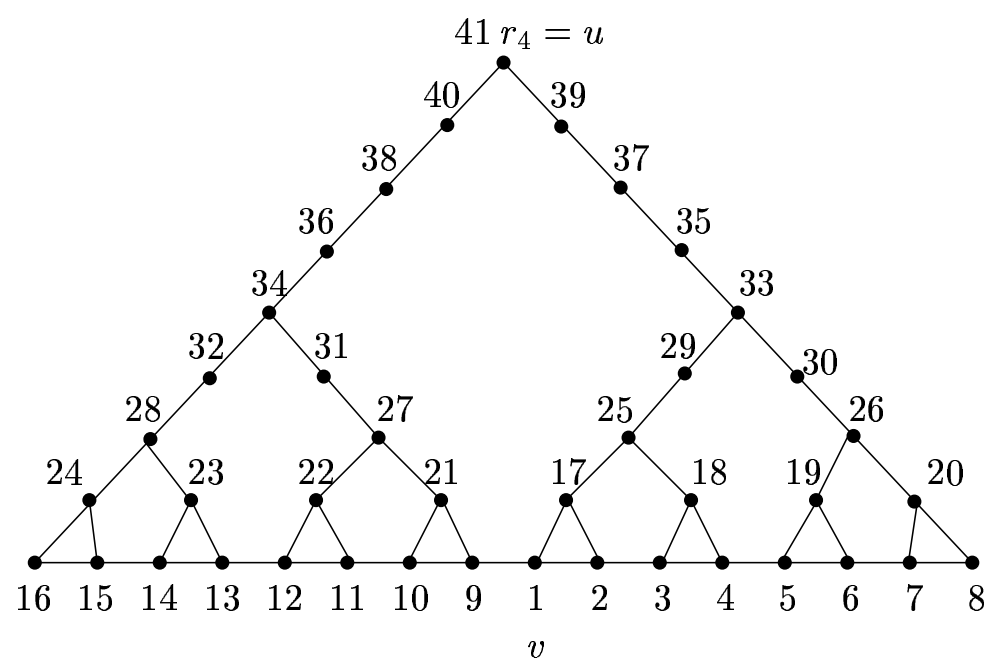

Figure 14: $G_{4}$ and the second LexBFS.

A second obvious question concerns the power of the 2-sweep algorithm on arbitrary graphs. Unfortunately, the answer again is negative. In particular, for any $i>1$, there is a graph $G_{i}$ where $\operatorname{ecc}(v)=\operatorname{diam}\left(G_{i}\right)-2^{i-1}+1$, where $v$ is the vertex returned by algorithm 2 . We construct $G_{i}$ as follows: Let $T_{1}$ be a 2-leaf tree with root $r_{1} . T_{i}, i>1$, is formed from two copies of $T_{i-1}$ by making $r_{i}$, the root of $T_{i}$, adjacent to the two $r_{i-1}$ roots. Each $r_{i} r_{i-1}$ edge then has $2^{i-2}-1$ new vertices inserted. Finally $G_{i}$ is formed from $T_{i}$ by creating a path on the leaves of $T_{i}$ in the obvious way. $G_{4}$ is shown in figure 13. If $w$ is the leftmost leaf of the right $T_{i-1}$ and the next vertex chosen in the LexBFS from $w$ is the rightmost leaf of the left $T_{i-1}$, then the LexBFS will end at $u=r_{i}$ (see figure 13). If the second LexBFS starts at $u$ and breaks ties by choosing the last eligible vertex in the previous sweep, then $v$, the last vertex, is the same as 
$w$ (see figure 14). It is easy to see that $e c c(v)=2^{i-1}$ and $\operatorname{diam}\left(G_{i}\right)=2^{i}-1$ as witnessed by the extreme leaves.

As a final comment, we note that the results in this paper add to the growing evidence of the similar roles played by LexBFS for chordal and AT-free graphs. It would be interesting to find a structural result to explain this surprising phenomenon.

\section{Acknowledgments}

D. C. wishes to thanks NSERC for financial assistance and LIRMM for their hospitality during his visit, and F.D.'s research was supported by the DFG. The authors would like to thank the anonymous referees for their helpful comments.

\section{References}

[1] D. Aingworth, C. Chekuri, P. Indyk, and R. Motwani, Fast estimation of diameter and shortest paths (without matrix multiplication), SIAM J. on Computing, 28 (1999), 1167-1181.

[2] A. BrandstäDt, V.D. Chepoi, and F.F. Dragan, The algorithmic use of hypertree structure and maximum neighbourhood orderings, Proceedings of WG'94 "GraphTheoretic Concepts in Computer Science", LNCS 903 (E.W. MAYR, G. SCHMIDT and G. Tinhofer, eds.) (1995) 65-80, Discrete Appl. Math. 82 (1998), 43-77.

[3] P. Buneman, A characterization of rigid circuit graphs, Discr. Math., 9 (1974), 205-212

[4] V.D. ChepoI, On distances in benzenoid systems, J. Chem. Inference Comput. Sci., 36 (1996) 1169-1172.

[5] V.D. Chepoi and F.F. Dragan, Disjoint sets problem, unpublished, 1992.

[6] D. Coppersmith and S. Winograd, Matrix multiplication via arithmetic progression, Proceedings of the 19th ACM Symposium on Theory of Computing, (1987), 1-6.

[7] D.G. Corneil, F.F. Dragan, M. Habib and C. Paul, Diameter Determination on Restricted Graph Families, Proceedings of WG'98 "Graph-Theoretic Concepts in Computer Science", LNCS 1517 (J. HromkoviC and O. SYKORA, Eds.), (1998) 192-202.

[8] D.G. Corneil, S. Olariu and L. Stewart, Linear time algorithms for dominating pairs in asteroidal triple-free graphs, SIAM J. on Computing, 28 (1999) 1284-1297.

[9] D.G. Corneil, S. Olariu and L. Stewart, Asteroidal triple-free graphs, SIAM Journal on Discrete Mathematics, 10 (1997), 399-431.

[10] E. Dahlhaus, Optimal (parallel) algorithms for the all-to-all vertex distances problem for certain graph classes, Proceedings of WG'92 "Graph-Theoretic Concepts in Computer Science", LNCS 657, (1993) 60-69.

[11] F.F. DRAgan, Dominating cliques in distance-hereditary graphs, Proceedings of SWAT'94 "4th Scandinavian Workshop on Algorithm Theory", LNCS 824 (E.M. SchmidT and S. SKYUm, Eds.), (1994) 370-381.

[12] F.F. Dragan, Almost diameter of a house-hole-free graph in linear time via LexBFS, Discrete Appl. Math., 95 (1999), 223-239.

[13] F.F. Dragan and F. NiCOLAI, LexBFS-orderings of distance-hereditary graphs with application to the diametral pair problem, Discrete Appl. Math., 98 (2000), 191-207.

[14] F.F. Dragan, F. Nicolai and A. BrandstäDt, LexBFS-orderings and powers of graphs, Proc. of the WG'96, LNCS 1197, 166-180, 1997. 
[15] M. FARBER, Characterizations of strongly chordal graphs, Discrete Mathematics, 43 (1983), 173-189.

[16] A.M. Farley and A. Proskurowski, Computation of the center and diameter of outerplanar graphs, Discrete Appl. Math., 2 (1980) 185-191.

[17] F. GavRIL, The intersection graphs of subtrees in trees are exactly the chordal graphs. J. Comb. Theory (B), 16 (1974), 47-56.

[18] F. GavRIL, A recognition algorithm for the intersection graph of directed paths in directed trees, Discr. Math., 13 (1975), 337-249.

[19] M.C. Golumbic, Algorithmic Graph Theory and Perfect Graphs, Academic Press, New York 1980 .

[20] K. Han, Chandra N. Sekharan and R. Sridhar, Unified all-pairs shortest path algorithms in the chordal hierarchy, Discrete Appl. Math. 77 (1997), 59-71.

[21] G. HANDLER, Minimax location of a facility in an undirected tree graph, Transportation Sci., 7 (1973), 287-293.

[22] B. Jamison and S. Olariu, On the semi-perfect elimination, Advances in Applied Math. 9 (1988), 364-376.

[23] C.G. LeKkerkerker and J.C. Boland, Representation of a finite graph by a set of intervals on the real line, Fundamenta Mathematicae, 51 (1962) 45-64.

[24] M. Lesk, Couplages maximaux et diamètre de graphes, $\mathrm{PhD}$ thesis, Université Pierre et Marie Curie, Paris 6, October 1984.

[25] S. Olariu, A simple linear-time algorithm for computing the center of an interval graph, Int. J. Comput. Math., 34 (1990), 121-128.

[26] C. PAUl, Parcours en largeur lexicographique: un algorithme de partitionnement, application aux graphes et généralisation, $\mathrm{PhD}$ thesis, University of Montpellier II, France, 1998.

[27] D. Rose, R.E. TARjan and G. Lueker, Algorithmic aspects on vertex elimination on graphs, SIAM J. Computing 5 (1976), 266-283.

[28] R. SEIDEL, On the all-pair-shortest-path problem, Proceedings of the 24th ACM Symposium on Theory of Computing, (1992), 745-749.

[29] R.E. TARJAn and M. YANnAKAKIS, Simple linear-time algorithms to test chordality of graphs, test acyclicity of hypergraphs, and selectively reduce acyclic hypergraph, SIAM J. Comput, (1984) 566-579. 